\title{
Pentraxin-2 suppresses c-Jun/AP-1 signaling to inhibit progressive fibrotic disease
}

\author{
Naoki Nakagawa, ${ }^{1,2,3}$ Luke Barron, ${ }^{4}$ Ivan G. Gomez, ${ }^{1,2,4}$ Bryce G. Johnson, ${ }^{1,2,4}$ Allie M. Roach, ${ }^{1,2,4}$ \\ Sei Kameoka, ${ }^{4}$ Richard M. Jack, ${ }^{5}$ Mark L. Lupher Jr., ${ }^{5}$ Sina A. Gharib, ${ }^{2,6,7}$ and Jeremy S. Duffield ${ }^{1,2,4}$ \\ 'Division of Nephrology, Departments of Medicine and Pathology, and 'Institute of Stem Cell and Regenerative Medicine, \\ University of Washington, Seattle, Washington, USA. ${ }^{3}$ Department of Internal Medicine, Asahikawa Medical University, \\ Asahikawa, Japan. ${ }^{4}$ Research and Development, Biogen, Cambridge, Massachusetts, USA. ${ }^{5}$ Promedior Inc., Lexington, \\ Massachusetts, USA. ${ }^{6}$ Computational Medicine Core, ${ }^{7}$ Divsion of Pulmonary and Critical Care Medicine, University of \\ Washington, Seattle, Washington, USA.
}

Pentraxin-2 (PTX-2), also known as serum amyloid P component (SAP/APCS), is a constitutive, antiinflammatory, innate immune plasma protein whose circulating level is decreased in chronic human fibrotic diseases. Here we show that recombinant human PTX-2 (rhPTX-2) retards progression of chronic kidney disease in $\mathrm{Col} 4 \mathrm{a} 3$ mutant mice with Alport syndrome, reducing blood markers of kidney failure, enhancing lifespan by $20 \%$, and improving histological signs of disease. Exogenously delivered rhPTX-2 was detected in macrophages but also in tubular epithelial cells, where it counteracted macrophage activation and was cytoprotective for the epithelium. Computational analysis of genes regulated by rhPTX-2 identified the transcriptional regulator c-Jun along with its activator protein-1 (AP-1) binding partners as a central target for the function of rhPTX-2. Accordingly, PTX-2 attenuates c-Jun and AP-1 activity, and reduces expression of AP-1dependent inflammatory genes in both monocytes and epithelium. Our studies therefore identify rhPTX-2 as a potential therapy for chronic fibrotic disease of the kidney and an important inhibitor of pathological c-Jun signaling in this setting.

Authorship note: N. Nakagawa and L. Barron contributed equally to this work.

Conflict of interest: R.M. Jack is the CSO, COO, and President of Promedior Inc. M.L. Lupher Jr. is a former member of Promedior Inc. J.S. Duffield holds stock options in Promedior Inc.

Submitted: March 8, 2016 Accepted: October 26, 2016 Published: December 8, 2016

Reference information: JCI Insight. 2016;1(20):e87446. doi:10.1172/jci.insight.87446.

\section{Introduction}

Pentraxin-2 (PTX-2), also known as serum amyloid P component (SAP or APCS), is a highly phylogenetically conserved, naturally circulating homopentameric plasma protein and a soluble pattern recognition receptor of the innate immune system (1-3). The unique binding activities of PTX-2 suggest that it may localize specifically to sites of injury and function to aid in the removal of damaged tissue, by non-phlogistic mechanisms. Genetic mutation of the PTX-2 gene in mice leads to autoimmunity and enhanced fibrosis, and is associated with impaired clearance of dying cells; the SAP/APCS gene locus has been associated with inflammatory disease, including juvenile rheumatoid arthritis, suggesting that endogenous PTX-2 may protect against inflammatory damage and autoimmunity (4-6). In chronic disease settings, circulating levels of PTX-2 in patients are lower than normal, suggesting that deficiency may contribute to disease $(2,7)$. The recent discovery that PTX-2 can regulate certain monocyte/macrophage differentiation states has identified one mechanism by which PTX-2 acts as a novel and potentially powerful antiinflammatory and antifibrotic agent (8). A fully recombinant form of the human PTX-2 protein, designated PRM-151, has recently been initiated in human clinical trials for fibrotic diseases of lung and bone marrow, where resolution of bone marrow fibrosis was accompanied by improved hematologic parameters $(9,10)$. Although PTX-2 binds to Fc $\gamma$ receptors and a new binding receptor, DC-SIGN, has recently been identified, the biochemical mechanisms by which PTX-2 inhibits inflammatory signaling have remained obscure $(8,11-15)$.

Chronic kidney disease (CKD) syndromes affect more than $10 \%$ of the population in developed countries (16) and have many causes, including hypertension, diabetes mellitus, vascular disease, infections, and xenobiotic toxicity, as well as rare genetic causes, including mutations in basement membrane proteins, exemplified by collagen type $\operatorname{IV}(\alpha) 3$. These diseases are characterized by glomerular and interstitial fibrosis, 
inflammatory cell recruitment, capillary destruction, and tubular injury leading to progressive loss of kidney function over months and years (17). Despite disparate causes, all CKDs show marked recruitment of inflammatory macrophages, which has been shown to contribute disease progression $(18,19)$.

We previously demonstrated that fibrogenesis in short-term models of kidney disease could be blocked by exogenous systemic recombinant rhPTX-2 (2). Here we determined whether rhPTX-2 treatment could also ameliorate the progressive loss of kidney function in mice with a chronic progressive kidney disease. Mice deficient for the kidney microvascular basement membrane protein collagen type $\operatorname{IV}(\alpha) 3\left(C_{0} / 4 a 3^{-1}\right.$ mice) exhibit key pathological features of human Alport syndrome, including advancing glomerulosclerosis and tubulointerstitial fibrosis, together with intense recruitment of inflammatory macrophages (20). Studying the effects of rhPTX-2 in this model permitted pertinent analysis of the efficacy and mechanisms of action of PTX-2 in the setting of chronic disease.

\section{Results}

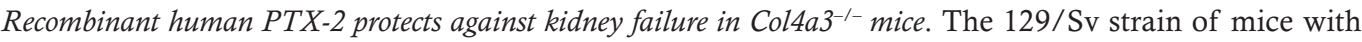
homozygous deletion mutation of the Col4a3 gene develops kidney disease as detected by albuminuria as early as 3 weeks of age $(20,21)$. The first histological signs of disease are seen by light microscopy in some glomeruli at 5 weeks, with occasional basement membrane thickening and increased matrix deposition in the capillary loop regions $(21,22)$. Glomerulosclerosis and tubulointerstitial fibrosis normally progress rapidly thereafter and result in organ failure and death by approximately 10 weeks of age. The distribution of rhPTX-2 at 9 weeks following administration of a twice-weekly intraperitoneal injection of rhPTX-2 was evaluated in diseased mice (Figure 1A). rhPTX-2 was concentrated in macrophages, as expected (Figure 1C, bottom row right, and Figure 1E), but was also readily identified in proximal tubules by colocalization with a specific proximal tubule marker, lotus tetragonolobus lectin (LTL) (Figure 1C, bottom row center, and Figure 1D). In previous studies, following ischemic kidney injury or mechanical ureteral obstructive kidney disease, rhPTX-2 was detected within the tubule lumen with nuclear debris, as well as binding to interstitial macrophages (2). Vehicle-treated mutant mice had substantial kidney failure by 9 weeks, but rhPTX-2 treatment preserved kidney function, with substantially lower levels of both blood urea nitrogen (BUN) and albuminuria (Figure 1, F and G). This outcome was reproduced in a separate cohort of mice also administered rhPTX-2 twice weekly to 9 weeks of age. Again, rhPTX-2 decreased plasma concentrations of BUN $(103.3 \pm 10.6$ vs. $72.4 \pm 8.4 \mathrm{mg} / \mathrm{dl} ; P<0.05)$ in plasma and urinary albumin excretion $(16.4 \pm 2.3$ vs. $9.4 \pm 1.7 \mathrm{~g} / \mathrm{g} \mathrm{Cr} ; P<0.05)$. Overall, more than 20 mice were treated with rhPTX-2 in 2 independent experiments with significant protection from kidney failure.

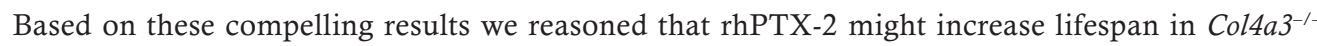
mice, which have a median survival time of $\sim 10$ weeks (23). Cohorts of mice were given rhPTX-2 (10 $\mathrm{mg} / \mathrm{kg}$ twice a week) or vehicle from week 3.5 until week 12 in an operator-blinded manner (Figure 1B). Vehicle-treated mice lost weight precipitously after 7 weeks, and all died by week 11 as a consequence of renal failure (Figure 1, H and I). By contrast, rhPTX-2 treatment delayed the onset of weight loss by 2 weeks and also increased median survival by 2 weeks, from day 63 to 77, equating to $>20 \%$ improvement in lifespan.

rhPTX-2 reduces glomerulosclerosis and tubulointerstitial kidney disease in Col4a3 ${ }^{-1-}$ mice. To understand the impact of rhPTX-2 on kidney disease progression in this model, histological parameters were evaluated in samples harvested at 9 weeks of age in an observer-blinded manner. Vehicle-treated $\mathrm{Col}_{4} \mathrm{a}^{-/-}$mice had typical extensive glomerulosclerosis (glomerular fibrosis with capillary destruction), the formation of glomerular crescents, and periglomerular fibrosis (Figure 2A, top row, center). Necrosis was not seen. rhPTX-2-treated mice had much less glomerulosclerosis: $30 \%$ of glomeruli showed no sclerosis, compared with only $15 \%$ in controls; and fewer than $15 \%$ of the glomeruli showed extensive sclerosis, as detected by the highest pathology score, compared with 30\% in control-treated mutants (Figure 2B). Because glomerulosclerosis is associated with a reduction in podocyte coverage of glomerular capillaries, podocyte number was measured by staining for the transcriptional regulator WT-1 (Figure 2A). rhPTX-2 administration preserved podocyte numbers in glomeruli (Figure 2C). Electron microscopy (EM) imaging of the capillary loops showed severe glomerular basement membrane (GBM) thickening, splitting, and lamellation in mutant mice with severe podocyte foot process effacement (Figure 2, A and D). By contrast, many capillary loops in rhPTX-2-treated mice showed small but classical basement membrane humps where

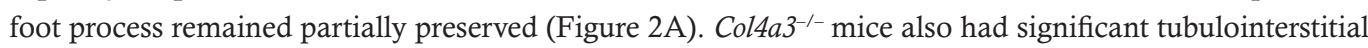


A

A Twice weekly i.p. rhPTX-2

Weeks 3

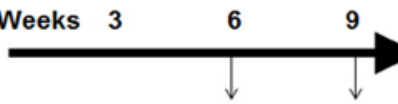

C

Analysis Analysis
B

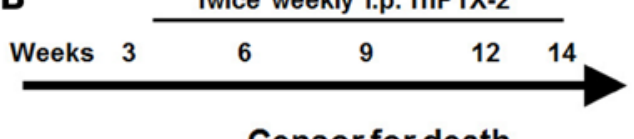

\section{$\mathrm{Col}_{4 a 3^{-/}+\mathrm{VEH}}$} Col4a $3^{-/}+$rhPTX-2
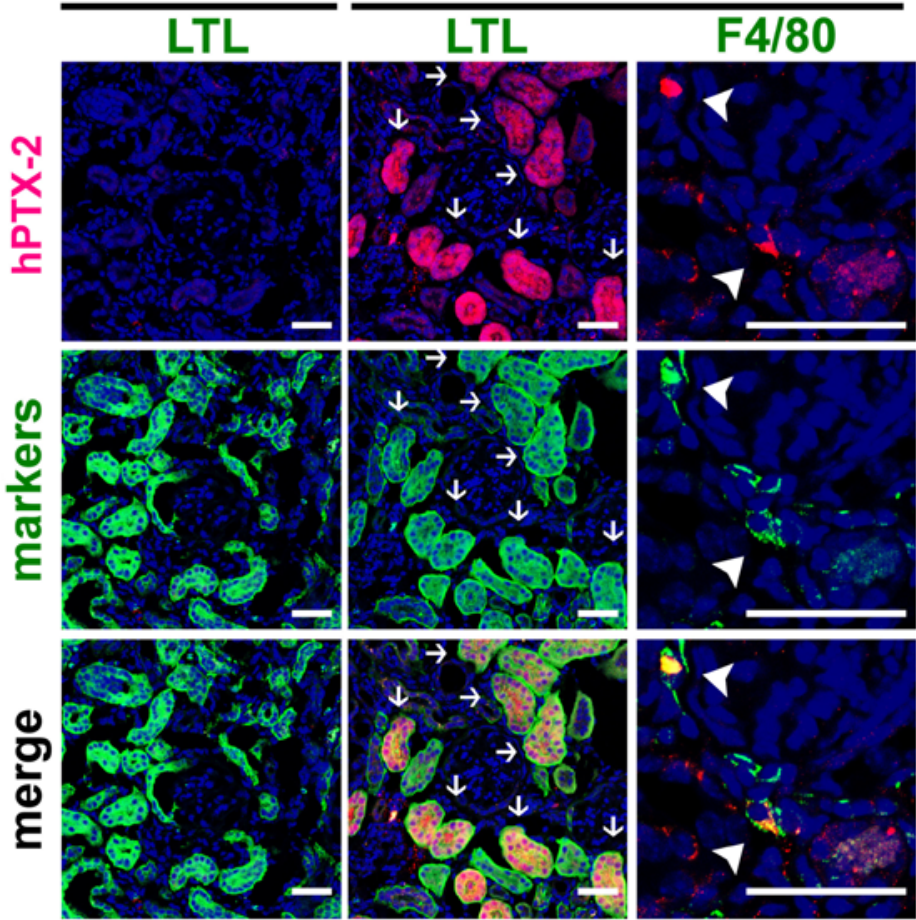

D

hPTX-2 / LTL

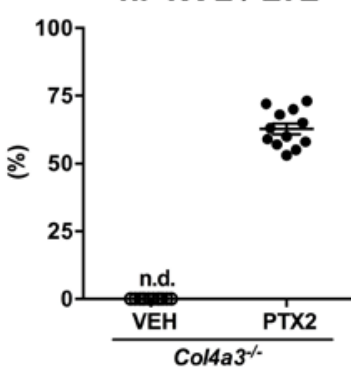

E

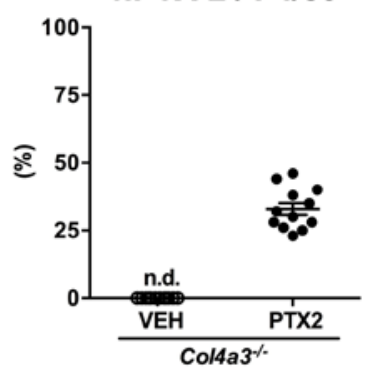

$\mathbf{F}$

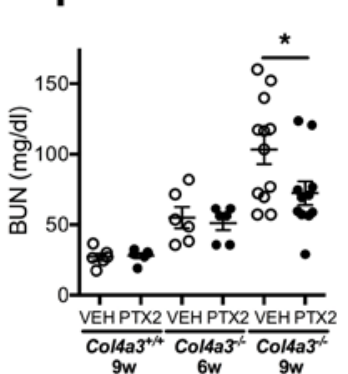

G

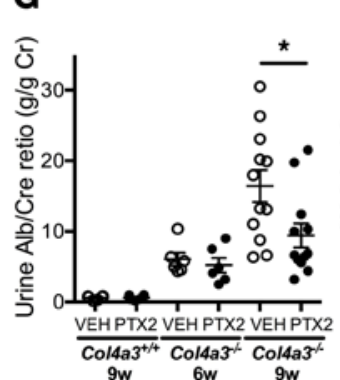

H

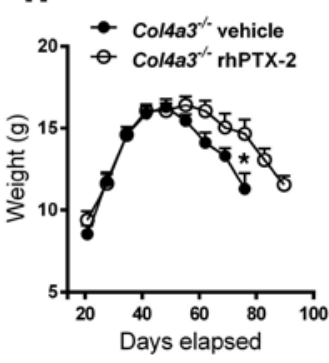

I

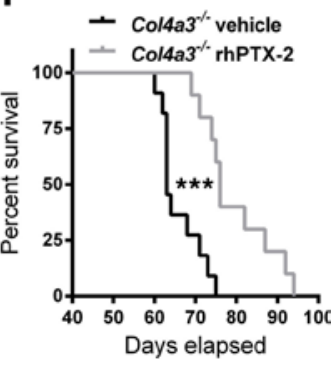

Figure 1. rhPTX-2 localizes to interstitial macrophages and proximal epithelial cells and protects $\mathrm{Col} \mathrm{a3}^{-/-}$mice from kidney disease progression. Experimental schema indicating rhPTX-2 delivery from 3.5 weeks after birth and (A) analysis at 42 days ( 6 weeks) and 63 days (9 weeks) or (B) continued to 94 days (14 weeks). (C) Split-panel confocal images of 9-week-old $\mathrm{Col}_{4 \mathrm{a} 3^{-/-}}$kidney cortex showing distribution of rhPTX-2 colocalized with proximal tubular epithelial cells (lotus lectin [LTL], arrows) and macrophages (F4/80, arrowheads). Scale bars: $50 \mu \mathrm{m}$. Proportions of (D) hPTX-2+LTL proximal tubular epithelial cells and (E) hPTX2+ $\mathrm{F} 4 / 80^{+}$macrophages at 9 weeks after twice weekly i.p. injections of rhPTX-2. (F) Plasma BUN levels at 9 weeks. (G) Urine albumin concentration normalized to urine creatinine at 9 weeks. (H) Body weights from start of treatment to death. (I) Kaplan-Meier survival curve. $n=6-12$ /group. ${ }^{*} P<0.05,{ }^{* * *} P<$ 0.001 (ANOVA with post hoc testing for multiple comparisons and Gehan-Breslow-Wilcoxon test for survival). VEH, vehicle; n.d., not detected.

pathology, characterized by interstitial fibrosis, tubular injury and atrophy, inflammation, and destruction of peritubular capillaries (Figure $3 \mathrm{~A}$ ). The extent of tubule injury was reduced $\sim 40 \%$ by rhPTX-2 administration (Figure 3, A and B), and Sirius red staining revealed that the extent of interstitial fibrosis was markedly attenuated (Figure 3, A and C). Therefore rhPTX-2 was highly protective against the development of glomerular, tubular, and interstitial disease. 
A

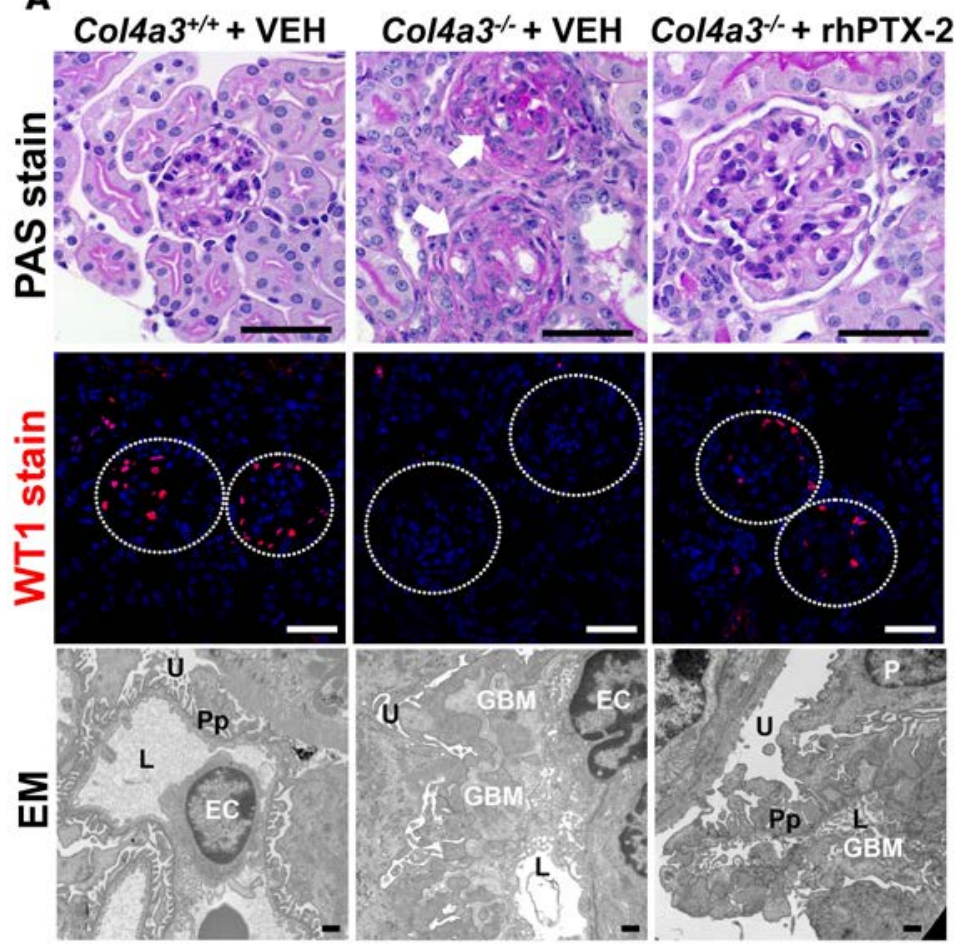

B

Glomerulosclerosis score

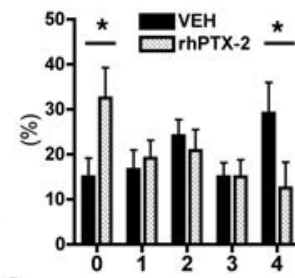

C
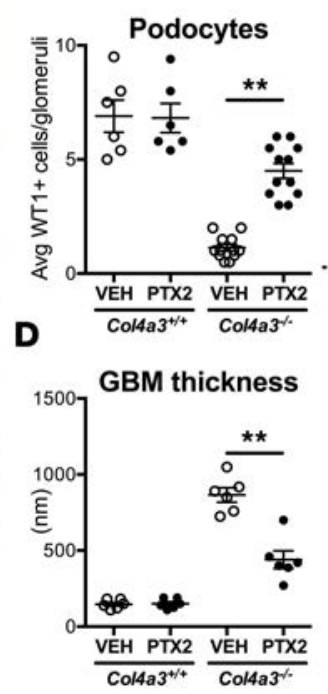

Figure 2. rhPTX-2 prevents glomerulosclerosis, glomerular basement membrane accumulation, and podocyte loss. (A) Representative images of kidney cortex at 9 weeks of age showing periodic acid-Schiff- stained (PAS-stained) images of glomeruli with glomerulosclerosis (white arrows); WT-1 immunofluorescence for podocytes within glomeruli (dotted lines); electron microscopy (EM) images of glomerular capillary loops showing severe glomerular basement membrane (CBM) thickening with humps, and podocyte effacement (loss of processes) in vehicle-treated Col4a3-1- mice. rhPTX-2-treated $\mathrm{Col} 4 a 3^{-1-}$ mice show areas of preserved basement membrane and partial foot process effacement, but small classical basement membrane humps persist. $\mathrm{U}$, urinary space; $\mathrm{P}$, podocyte; Pp, podocyte processes; L, capillary lumen; EC, endothelial cell. (B) Glomerulosclerosis score ranged from none (0) to $>75 \%$ of glomeruli affected (4). (C) WT-1+ podocytes per glomerular cross-section. (D) GBM thickness. Scale bars: $50 \mu \mathrm{m}$ (light) or $500 \mathrm{~nm}$ (EM). $n=6-12$ /group. ${ }^{*} P$ $<0.05$, ${ }^{* *} P<0.01$ (ANOVA with post hoc testing for multiple comparisons). VEH, vehicle.

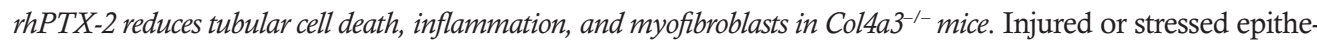
lial cells often die by apoptosis. rhPTX-2 reduced renal tubule cell apoptosis (Figure 4, A and B), as detected by the TUNEL method. Inflammation and fibrosis likely occur as a response to capillary and tubular injury. rhPTX-2 significantly reduced $\mathrm{F} 4 / 80^{+}$macrophages, as well as $\mathrm{CD} 11 \mathrm{~b}^{+}$leukocytes in general and within glomeruli (Figure 4, A, C, D, and F). Consistent with the reduction in fibrosis, kidney interstitial myofibroblasts detected by $\alpha$-SMA staining were significantly reduced in area (Figure $4, \mathrm{~A}$ and $\mathrm{E}$ ), and this was matched by decreased transcription of Acta2 (encoding $\alpha$-SMA) and fibrillar collagen genes such as Colla1 (Figure 4, G and $\mathrm{H})$. Finally, closely associated with the fibrotic process, rhPTX-2 preserved peritubular capillary (PTC) integrity (Figure 4I). Overall, rhPTX-2 reduced inflammation, tubular and capillary destruction, and fibrogenesis.

rhPTX-2 reduces the presence of M1 macrophages. PTX-2 has been reported to reduce M1 macrophage activation and stimulate antiinflammatory M2 type (Mreg) macrophages (2, 5, 24, 25). We therefore evaluated macrophage populations in glomeruli and the interstitium. Overall macrophage numbers in the diseased kidneys were reduced, and specifically, M1 macrophages labeled by coexpression of CD11b and CD86 were markedly reduced (Figure 5, A-C). By contrast, rhPTX-2 did not change the percentage of M2-like, CD206 ${ }^{+}$macrophages, although the absolute number was reduced (Figure 5, A, D, and E). Additional measures of macrophage phenotypes were consistent with a loss of M1-type activated macrophages in this disease model, including decreased Trem1, Tnfa, Nos2, and Il6 transcriptional levels (Figure 5, F and G, and Supplemental Figure 1; supplemental material available online with this article; doi:10.1172/jci.insight.87446DS1). rhPTX-2 also reduced CSf1, a key growth factor for macrophages in non-lymphoid organs (data not shown). Yet apart from IL4ra, other markers that may indicate M2-like activation were unaffected, including $\operatorname{Arg} 1$ (Figure 5H), Igfr2, Fcrls (MSR2), Slamf1 (CD150), Wnt7b, CX3crl, and Igf1. Treatment with rhPTX-2 showed a trend toward increased Illo levels in whole kidney tissue, consistent with enhanced Illo production per macrophage from a smaller population of cells (Figure 5I). Overall these results suggest that PTX-2 limits total macrophage numbers, and limits M1-type macrophage activation, and potentially increases macrophage IL10 production in CKD. 
A $\mathrm{Col}_{4 a 3^{+/+}+\mathrm{VEH}}$
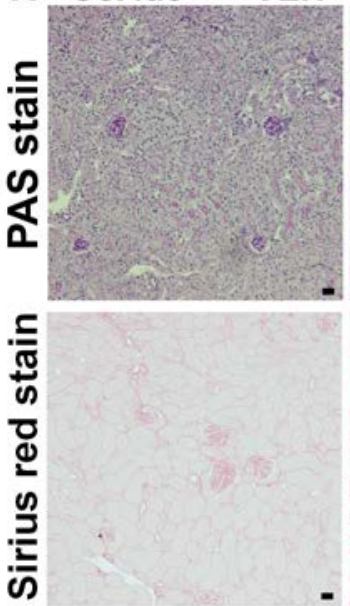

$\mathrm{Col}_{4} 3^{-/}+\mathrm{VEH}$
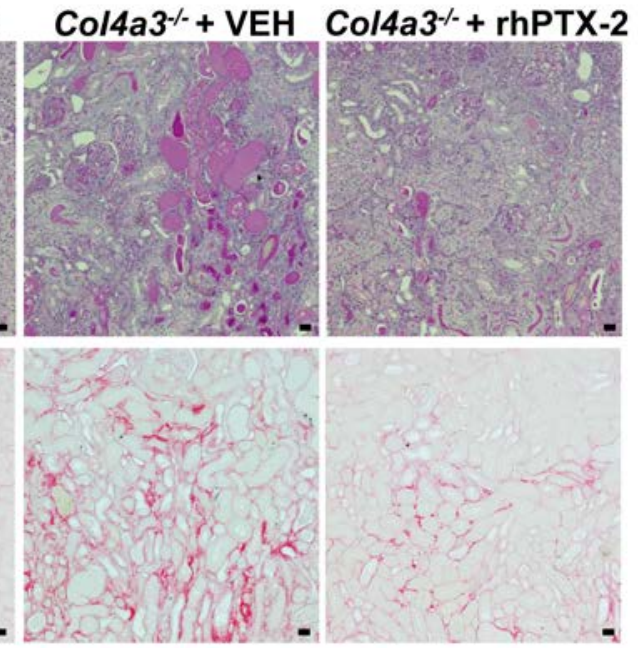

B

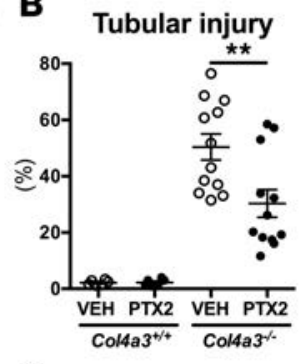

C

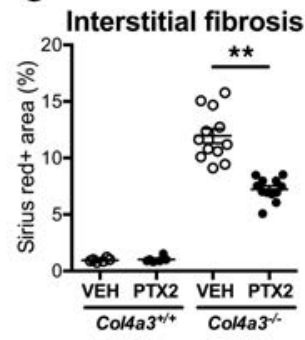

Figure 3. rhPTX-2 prevents tubular epithelial injury and interstitial fibrosis in Col4a3 ${ }^{-/-}$kidneys. (A) PAS-stained and Sirius red-stained images of kidney cortex. Note severe tubular injury with proteinaceous casts and loss of polarity and brush border of epithelium in Col4a $3^{-1-}$ mice (upper center panel). These pathological features are much milder following rhPTX-2 treatment. (B) Tubular injury score (C) Morphometric analysis of interstitial fibrosis detected by Sirius red staining. Scale bars: $50 \mu \mathrm{m}$. $n$ $=6-12$ /group. ${ }^{* *} P<0.01$ (ANOVA with post hoc testing for multiple comparisons). VEH, vehicle.

Gene set enrichment and protein network analyses identify c-Jun as a central regulator of PTX-2 function. To gain deeper insight into the role of PTX-2 in modulating Alport nephropathy, we assessed the effects of rhPTX-2

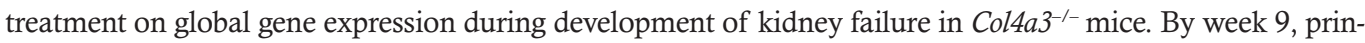

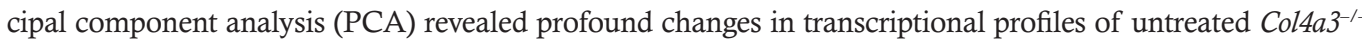

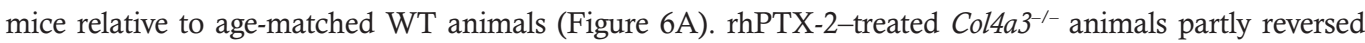
their position along the principal axes, implying that therapy reduced deviation from a healthy homeostatic state. PTX-2 causes pleiotropic changes in innate immunity by mechanisms that are not deeply understood. Therefore, we applied gene set enrichment analysis (GSEA) $(26,27)$ to identify with greater power pathways of coordinately regulated genes activated or suppressed during progression of kidney disease in $\mathrm{Col}_{4 \mathrm{a} 3^{-/-}}$mice and to assess whether these processes were targeted by rhPTX-2. We found pathways comprising upregulated genes were primarily involved in immunity, inflammation, and tissue remodeling, whereas downregulated genes were enriched in mitochondrial and metabolic processes (Figure 6B, Supplemental Figure 2, and Sup-

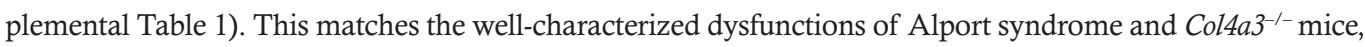
in which breakdown of multiple basic cellular processes combined with inflammation causes kidney failure (21). Strikingly, treatment with rhPTX-2 partly restored altered transcriptional profiles to their uninjured baseline across these disparate biological modules, highlighting a multifaceted therapeutic role for this molecule (Figure 6B and Supplemental Figure 3). Interestingly, rhPTX-2 created a different pattern of gene expression in extracellular matrix organization and the activator protein-1 (AP-1) pathways. Rather than partly reversing the effects of disease, here rhPTX-2 both overrode the disease phenotype and decreased expression of the genes within these modules to below that of healthy controls.

We noted that the individual genes most suppressed by rhPTX-2 treatment included a substantial number of kinases and transcription factors prominent in innate immunity (Supplemental Figure 3). We therefore performed a leading-edge analysis of these GSEA results to measure which genes made the greatest contribution to the pathways' enrichment signals, i.e., to evaluate which genes were most accountable for the protective effects of rhPTX-2 treatment (26). This computation likewise identified key components in inflammatory signaling pathways, including AP-1 (c-Jun, JunB, JunD, Fos12), MAPK (p38, Erk, MAPKAPK2 and -3, Tab2, MAP3K3,

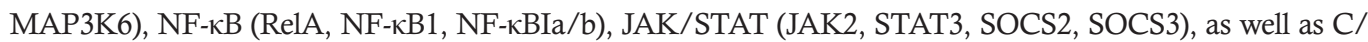
EBP $\beta$, TRAF6, and MyD88 (Supplemental Table 2). As with GSEA, pathway enrichment analysis using the GeneGo knowledge base also identified changes across diverse pathway maps representing inflammation, cellular distress, and tissue remodeling, and confirmed that members of the AP-1, MAPK, and NF- $\mathrm{BB}$ families were widely involved elements for the genes downregulated by rhPTX-2 (Supplemental Figure 4 and Supplemental Table 3). Intriguingly, a protein network analysis by Search Tool for the Retrieval of Interacting Genes/Proteins (STRING) (28) predicted that many of these suppressed factors formed an interrelated network, centered near c-Jun and ringed with other transcription factors that either are also AP-1 family members (Jun-B, Fos12) or form

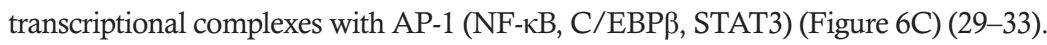


A

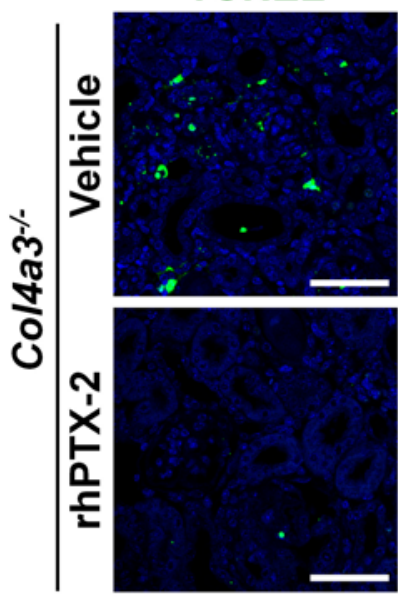

B

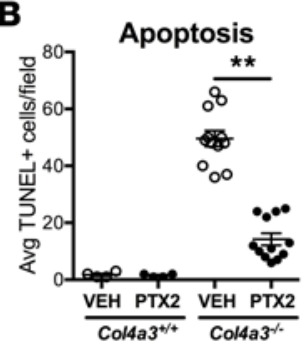

$\mathbf{F}$

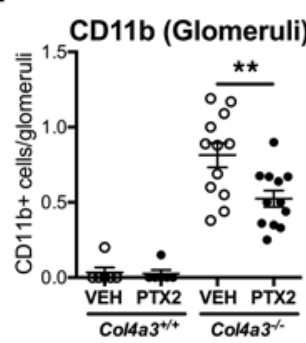

$\mathrm{F} 4 / 80$
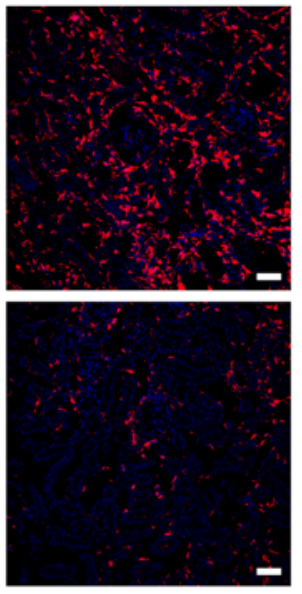

C

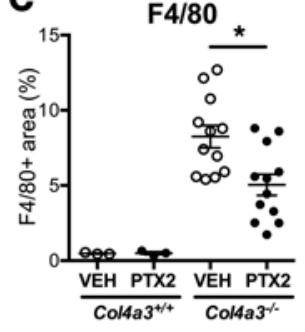

G

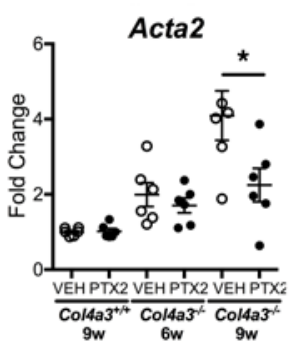

CD11b
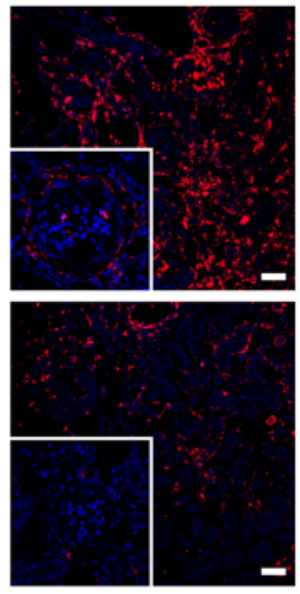

D

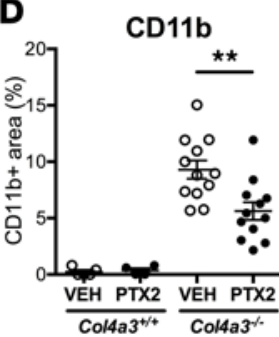

H

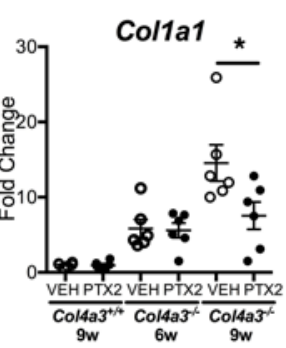

$\alpha$ SMA

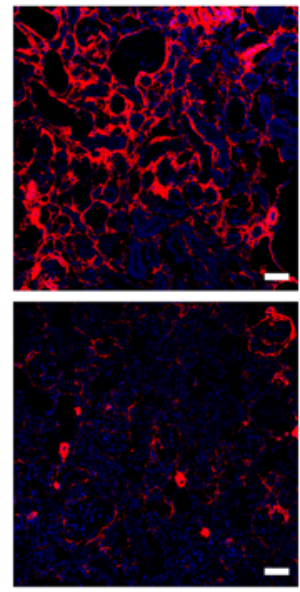

E Myofibroblasts

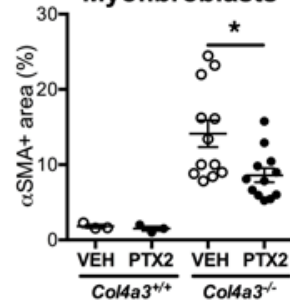

I

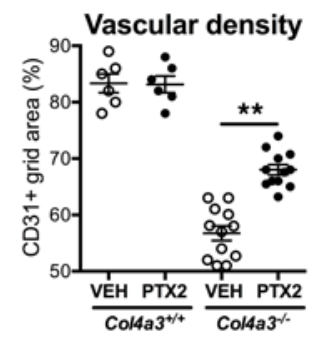

Figure 4. Epithelial cell death, myofibroblasts, and macrophage cell expansion are all attenuated by rhPTX-2. (A) Images of apoptotic tubular epithelial cells (TUNEL+), macrophages $\left(\mathrm{F} 4 / 80^{+}\right)$, myeloid cells (CD11 b $)$, and myofibroblasts $\left(\alpha-\mathrm{SMA}^{+}\right)$in kidney at 9 weeks. Insets show a glomerulus labeled for CD11b expression. (B) Tubular apoptosis, (C) macrophages, (D) myeloid cells, and (E) myofibroblasts. (F) CD11b+ cells per glomerular tuft. (G and $\mathbf{H})$ Q-PCR measuring Acta2 and Col1a1 transcripts in whole kidney tissue. (I) Quantification of peritubular capillary density. Scale bars: $50 \mu \mathrm{m} . n=$ 6-12/group. ${ }^{*} P<0.05,{ }^{* *} P<0.01$ (ANOVA with post hoc testing for multiple comparisons). Avg, average; VEH, vehicle.

In support of these findings from bioinformatics analyses, in whole kidney tissue we found that rhPTX-2 reduced c-Jun protein in both its total and activate phosphorylated states (Figure 6D; see complete unedited blots in the supplemental material). By contrast, rhPTX-2 increased C/EBP $\beta$, a factor regulated cooperatively by AP-1 and reported to play an important role in M2/Mreg reparative macrophage differentiation (34).

To test whether rhPTX-2 directly alters signaling through c-Jun and the AP-1 pathway, we developed a robust assay of human induced pluripotent stem cell-derived (iPSC-derived) monocyte activation with TNF- $\alpha$. rhPTX-2 treatment negatively regulated c-Jun in two ways: reducing MAPK-dependent phosphorylation at a crucial transcriptional activation site (Ser63); and causing total c-Jun protein levels to drop instead of rise (Figure 7, A and B). To investigate this suppressive effect further, we generated two different AP-1 transcriptional activity reporter assays in THP-1 monocytes. In one, dual AP-1/ NF-kB binding sequences expressed secreted alkaline phosphatase in MyD88-deficient cells (THP1-XBlue-defMyD). These monocytes showed low baseline activity and robust induction of the reporter in response to TNF- $\alpha$ that was suppressed in a dose-dependent manner by rhPTX-2 (Figure 7C). To confirm specificity, a c-Jun small molecule inhibitor, SR-11302, likewise suppressed the TNF- $\alpha$-induced signal. In the other assay, WT THP-1 monocytes 
A

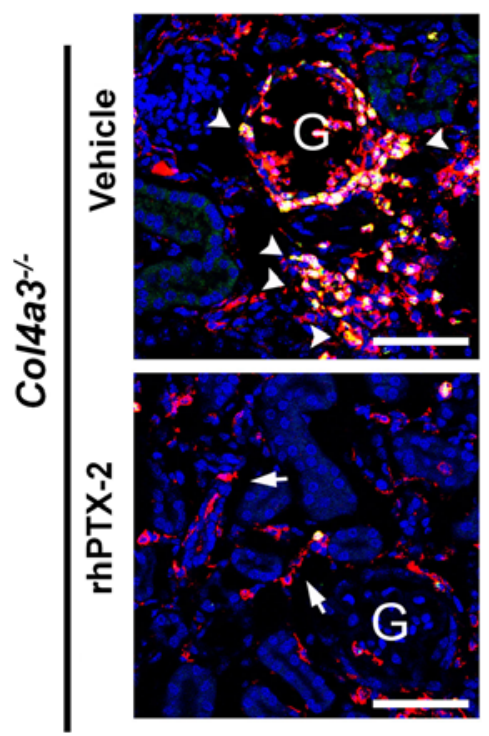

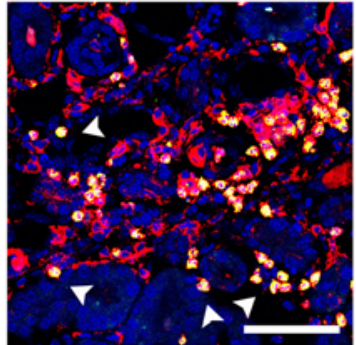

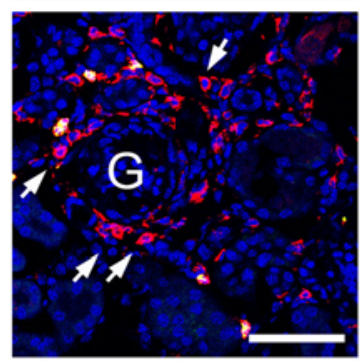

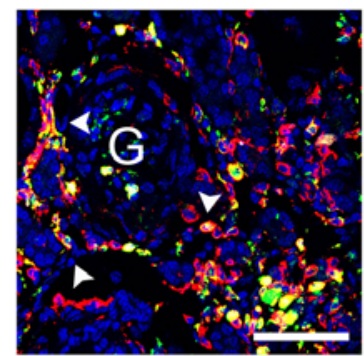
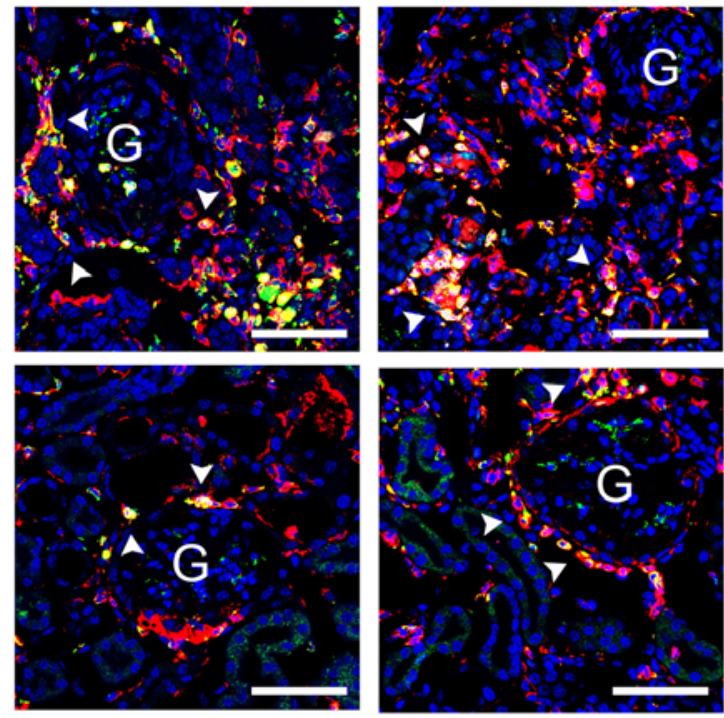

E
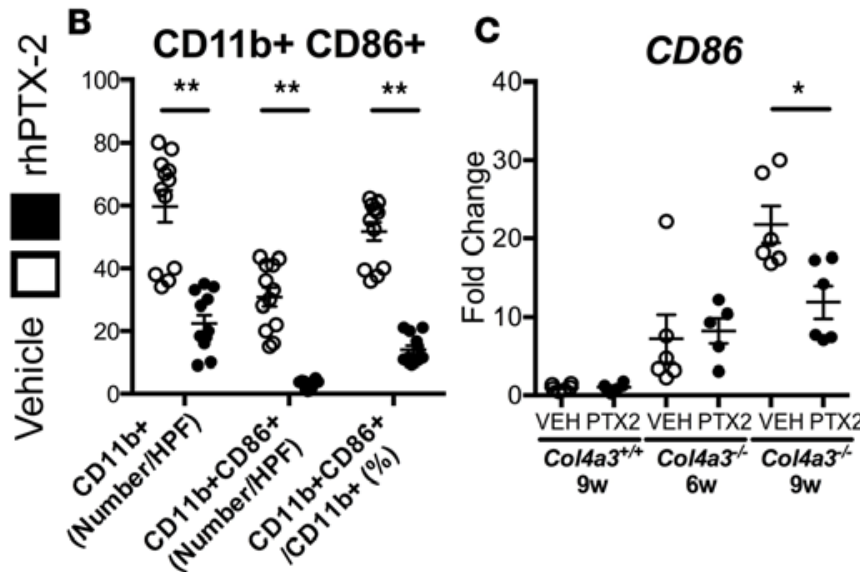

D

CD11b+CD206+
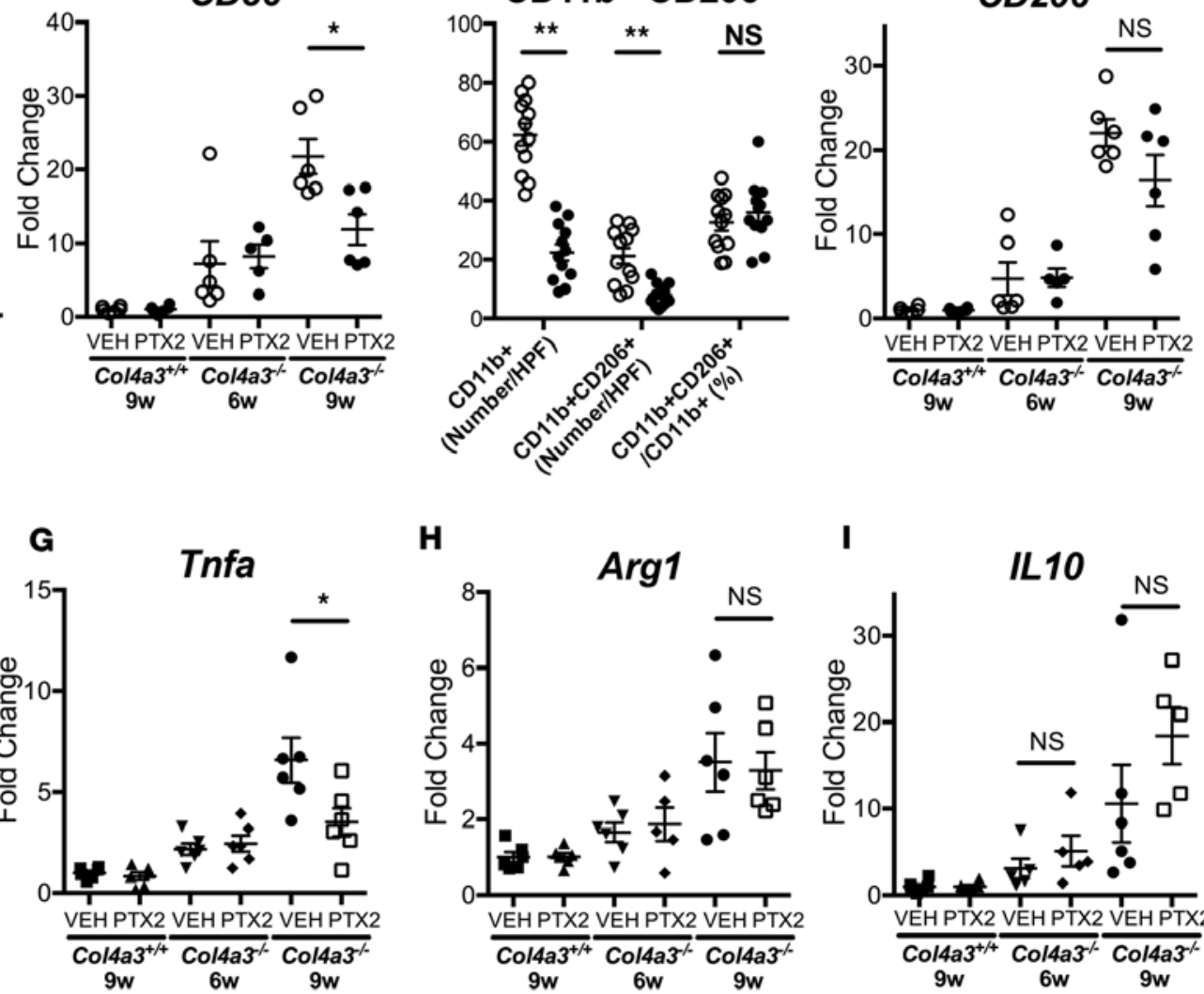

H

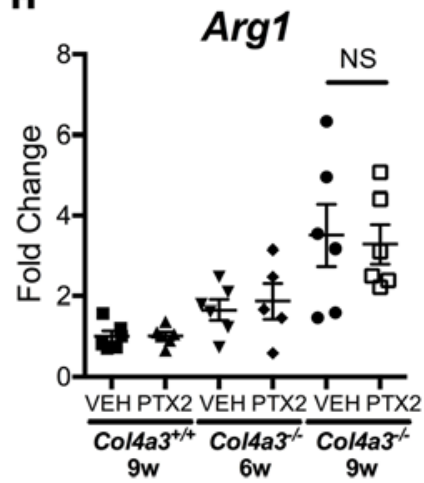

I

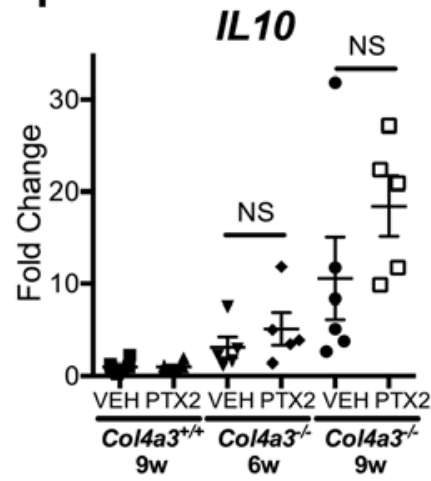

Figure 5. rhPTX-2 reduces the population of M1-type activated macrophages in Col4a3-1- mice. (A) Images of kidney M1 (CD86+) and M2 (CD206/mannose receptor ${ }^{+}$) macrophages at 9 weeks. G, glomerulus. (B and D) Numbers and percentages of CD86 ${ }^{+}$or CD206 ${ }^{+}$macrophages in tissue sections. (C and E-I) Q-PCR measuring M1-type (CD86, Trem1, Tnfa) and M2-like (CD206, Arg1) markers, and Il10 in whole kidney. Scale bars: $50 \mu \mathrm{m} . n=6-12 / g r o u p .{ }^{*} P<0.05$, ${ }^{* *} P<0.01$ (ANOVA with post hoc testing for multiple comparisons). VEH, vehicle. 


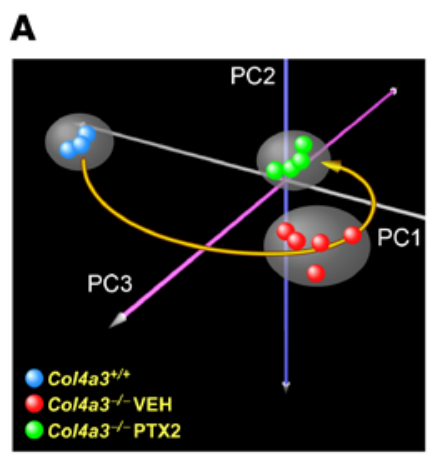

B

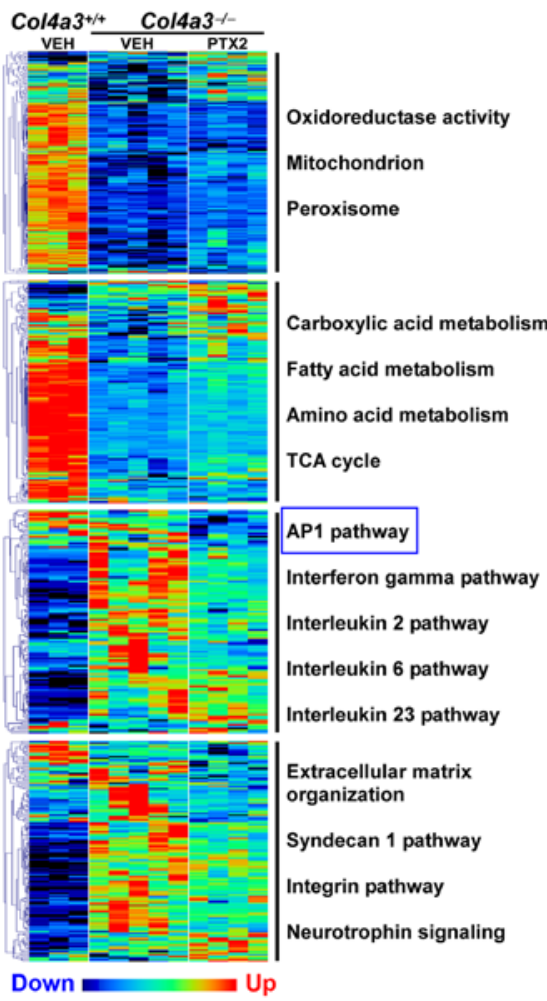

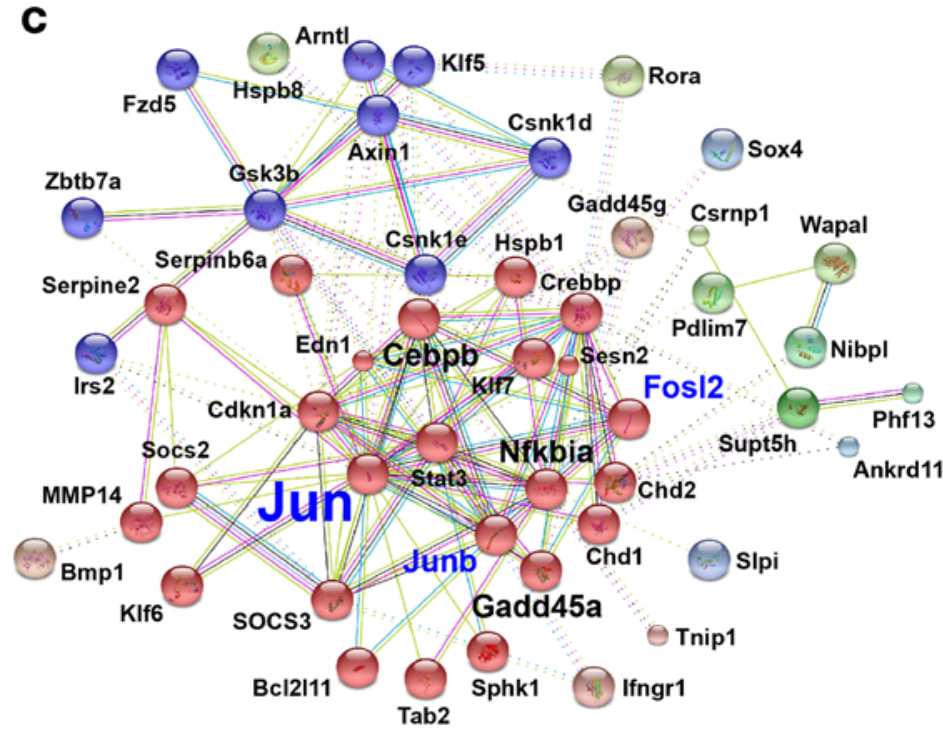

D

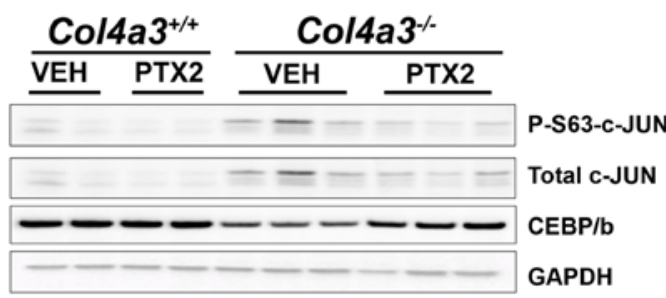

Figure 6. Gene set enrichment analysis identifies attenuated c-Jun signaling as a direct and central consequence of PTX-2 treatment. (A) Principal component analysis of kidney transcriptome. Treatment of $\mathrm{Col}_{4} a 3^{-/-}$mice with rhPTX-2 partly reverses separation from the uninjured $\mathrm{Col} 4 \mathrm{a} 3^{+/+}$animals, indicating rhPTX-2 therapy reduced transcriptional perturbation. (B) Summary of pathway analysis (CSEA) depicted as heatmaps of individual leading-edge genes (rows) and individual mice (columns). Enriched gene sets fell into 4 broad functional categories induced by disease: mitochondrion/oxidoreductase

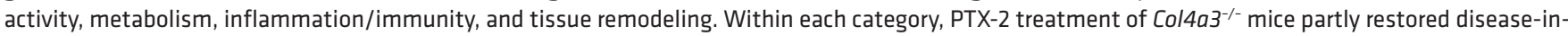
duced gene expression changes to their unperturbed baseline. Note only AP-1 signaling (blue box) was suppressed below baseline levels by rhPTX-2. (C) Abbreviated protein-protein interaction network predicted by STRING analysis of the top 100 leading-edge genes identified by GSEA and downregulated by rhPTX-2 treatment. Line color denotes type of evidence: green: neighborhood, red: gene fusion, blue: co-occurrence, black: coexpression, purple: experiments, cyan: databases, yellow: text mining, and lilac: homology. (D) Western blots showing the effect of disease and PTX-2 treatment on p-S63-c-Jun, total c-Jun, and C/EBP in kidney tissue. VEH, vehicle.

were stably transduced by lentivirus with a minimal CMV promoter and tandem AP-1 response elements strictly controlling luciferase transcription. Similarly, in these cells TNF- $\alpha$ induced AP-1 transcriptional activity, and this was suppressed by rhPTX-2 with dose dependency (Figure 7D). In keeping with these observations, stimulation of cultured primary human monocytes with rhPTX-2 increased IL-10 production while strongly downregulating other secreted factors associated with fibrosis (Figure 7E). These included IL-1R $\alpha$, CCL22, and PAI-1, where gene transcription depends on c-Jun and AP-1 promoter binding sites.

We also observed that rhPTX-2 regulated RelA (NF- $\mathrm{kB}$ p65), MyD88, and Traf6, integral factors in many cellular processes, including the Toll-like receptor signaling pathways in vivo (Supplemental Figure 4 
A

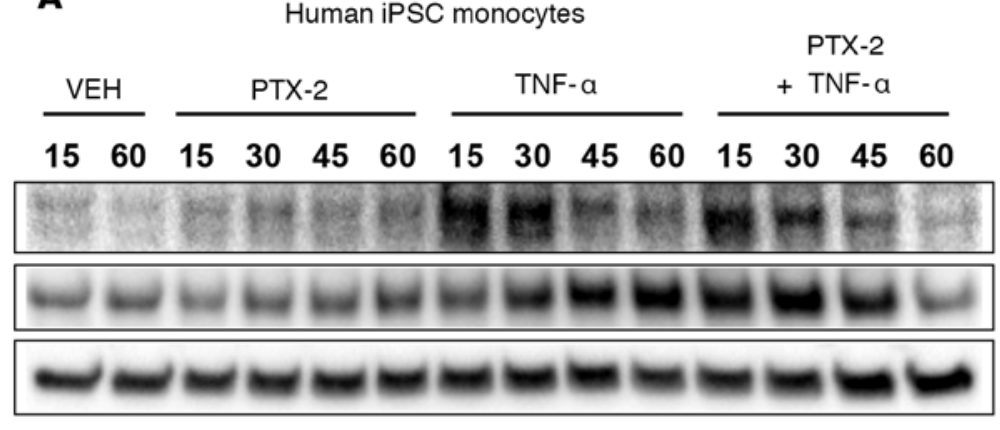

$$
\text { C } \quad A P-1 / N F k B \text { promoter activity }
$$

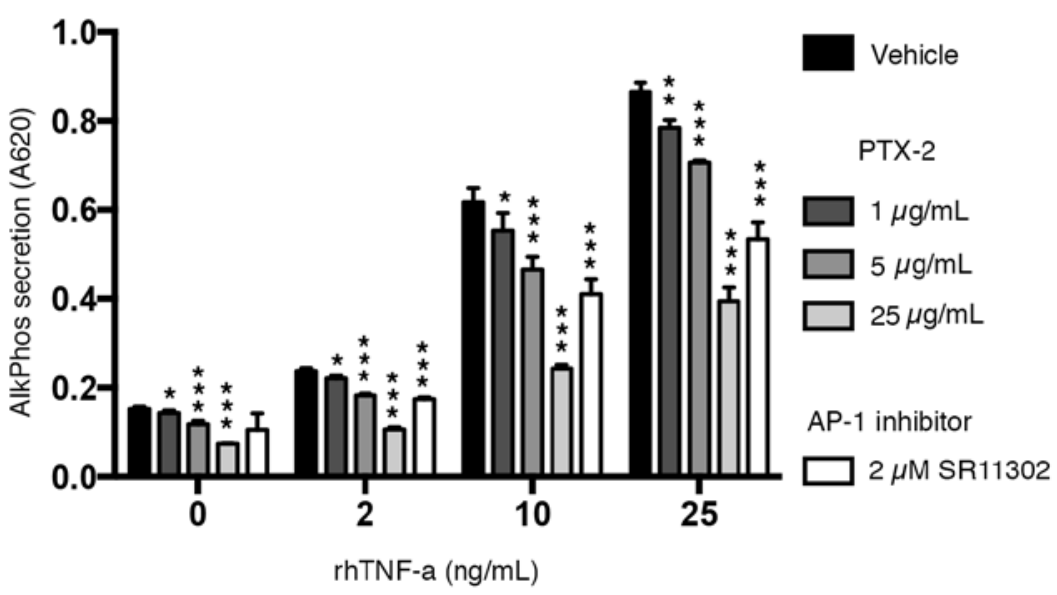

D AP1 promoter activity

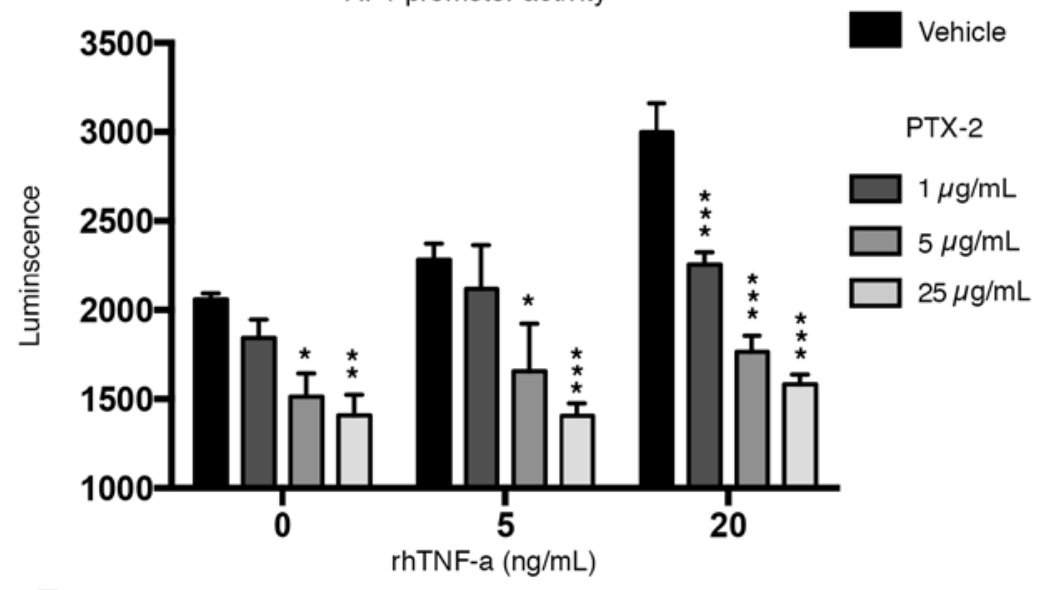

E

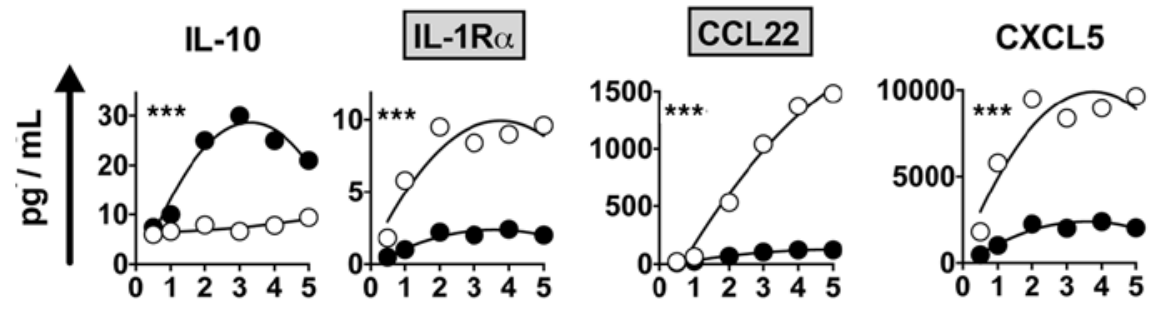

Days
B
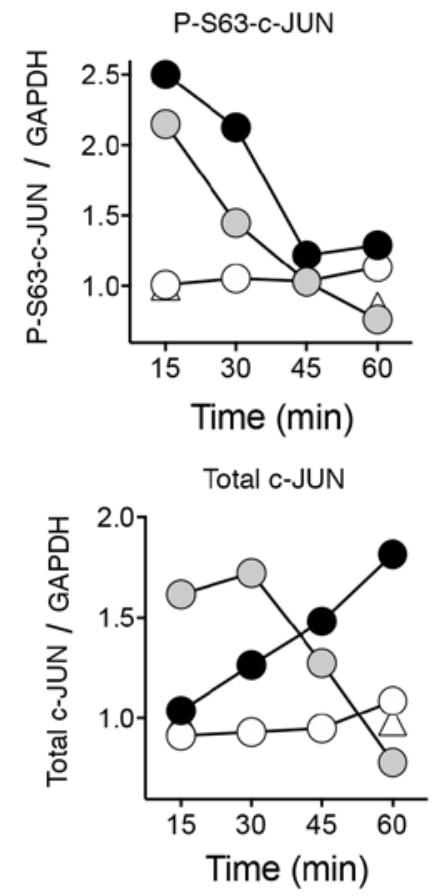

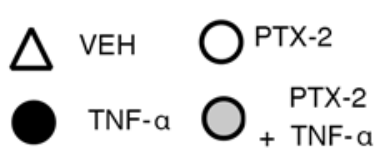


Figure 7. rhPTX-2 directly attenuates c-Jun and AP-1 complex signaling in human monocytes. (A and B) Time-course Western blots (A) and densitometry quantification (B) showing c-Jun phosphorylation (p-S63-c-Jun) and total c-Jun levels from 15 to 60 minutes after rhPTX-2 and/or TNF- $\alpha$ stimulation in human iPSC-derived monocytes, normalized to GAPDH. Note that rhPTX-2 attenuates both total and p-c-Jun in response to TNF- $\alpha$. Data represent 3 experiments. (C) Effect of concentrations of PTX-2 or AP-1 inhibitor SR11302 on TNF- $\alpha$-induced AP1/NF- $\mathrm{KB}$ promoter activity in human monocytes. (D) Effect of concentrations of PTX-2 on TNF- $\alpha$-induced AP-1 promoter activity alone, in human monocytes. (E) rhPTX-2-induced changes in the secretion of factors associated with fibrosis by primary human monocytes cultured from 12 to 120 hours. Presence of c-Jun and AP-1 binding sites in the promoters of these genes is indicated, based on the GeneCards database. $n=3-8$ /group $\left({ }^{*} P<0.05,{ }^{* *} P<0.01,{ }^{* *} P<0.001\right.$ (ANOVA with post hoc testing for multiple comparisons). VEH, vehicle; AlkPhos, alkaline phosphatase.

and Supplemental Table 2). Immunostaining of kidney tissue confirmed a reduction in total and nuclear-localized RelA in rhPTX-2-treated mice, in keeping with an overall reduction in inflammation and NF- $\mathrm{kB}$ signaling (Supplemental Figure 5, A and B). However, and in contrast to the c-Jun pathway, rhPTX-2-treated iPSC-derived monocytes stimulated with TNF- $\alpha$ showed similar RelA activation (Supplemental Figure $5, \mathrm{C}$ and $\mathrm{D}$ ), suggesting that rhPTX-2 reduced NF- $\mathrm{BB}$ signaling indirectly, as a secondary effect to suppressing AP-1 and potentially other pathways.

Collectively, these results demonstrate that the $\mathrm{Col}_{4} 3^{-/-}$animal model of Alport nephropathy shares common pathological characteristics with other CKDs such as diabetic nephropathy $(35,36)$, including dysregulation of immunoinflammatory responses and metabolic and mitochondrial dysfunction. Whole tissue analysis indicates that rhPTX-2 has broad effects on the kidney, rather than inhibiting or activating a single receptor-mediated signaling pathway, but that a major effect of PTX-2 is on the direct modulation of AP-1 transcriptional regulation.

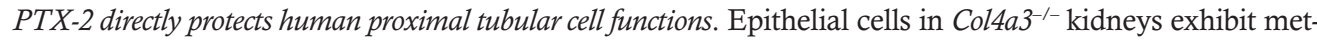
abolic and mitochondrial distress, and interventions targeting these pathways ameliorate disease (18, 19, 21, 27, 35-37). Because rhPTX-2 treatment also reduced changes in metabolic and mitochondrial pathways, preserved epithelial functions, and colocalized in vivo with epithelial cells (Figure 1, C and D), we hypothesized that PTX-2 may exert beneficial functions directly on the tubule epithelium beyond its effects on macrophages.

Epithelial cells do not have Fc $\gamma$ Rs, the receptor complexes by which PTX-2 binds to and mediates its antiinflammatory effects in macrophages $(2,38)$, but we reasoned that the epithelium may instead have a different PTX-2 receptor. To test this, human primary proximal tubule epithelial cells (PTECs) were purified and cultured in conditions to preserve their phenotype. PTEC monolayers were incubated with rhPTX-2 briefly at $4^{\circ} \mathrm{C}$, to detect cell surface binding in the absence of any serum factors that might indirectly connect rhPTX-2 to epithelial cells. Following washing, the protein was detected on the cell surface (Figure 8A). Such binding clustered in regions and was not uniform, perhaps reflecting cell polarity. The experiment was repeated at $37^{\circ} \mathrm{C}$. rhPTX-2 was now rapidly detected in intracellular compartments (Figure $8 \mathrm{~A}$ ). Costaining suggested that some rhPTX-2 was internalized within clathrin-associated intracellular vesicles, while it was not detected in lysosomes or in caveolae

PTECs that had bound and internalized rhPTX-2 displayed attenuated c-Jun signaling when shocked by plasma (Figure 8, B and C). Activating phosphorylation at a key MAPK target site (c-Jun Ser63) was more strongly inhibited by rhPTX-2 in PTECs than in iPSC-derived monocytes (compare to Figure 7, A and B), while total levels of c-Jun were slightly elevated by plasma shock and not altered by rhPTX-2 (Figure $8, \mathrm{~B}$ and $\mathrm{C}$ ). This effect on active c-Jun was recapitulated in an assay of nuclear accumulation of $\mathrm{p}-\mathrm{C}$ JUN in response to plasma shock (Figure 8D), confirming a direct effect of PTX-2 on AP-1 signaling in the epithelium. Exposure to plasma caused significant transcriptional responses in PTECs, stimulating pathways involved in remodeling, inflammation, and apoptosis while suppressing oxidative phosphorylation, ribosomal, and mitochondrial function (Supplemental Figure 6). These transcriptional patterns largely mirrored our findings in diseased kidney tissue from the $\mathrm{Col}_{4} \mathrm{a}^{-1-}$ model of Alport nephropathy (Figure 6B). Treatment of PTECs with rhPTX-2 suppressed the effects of plasma on each of these functional pathways (Supplemental Figure 6), indicating that a major additional beneficial effect of rhPTX2 treatment in vivo may be direct activity on the proximal tubule.

To test the impact of rhPTX-2 on PTEC responses at a functional level and model the milieu induced by kidney disease, PTECs were exposed to plasma or a cytokine implicated in epithelial injury, TGF- $\beta 1$. In both settings this stimulus triggered major phenotypic changes to PTECs, including loss of junctional E-cadherin and the de novo expression of the intermediate filament protein vimentin (Figure 8E), both distinct features associated with remodeling. Coincubation with $25 \mu \mathrm{g} / \mathrm{ml} \mathrm{rhPTX}-2$ abrogated these changes. Similarly, exposure of PTECs to plasma or TGF- $\beta 1$ stimulated a marked increase in release of mito- 

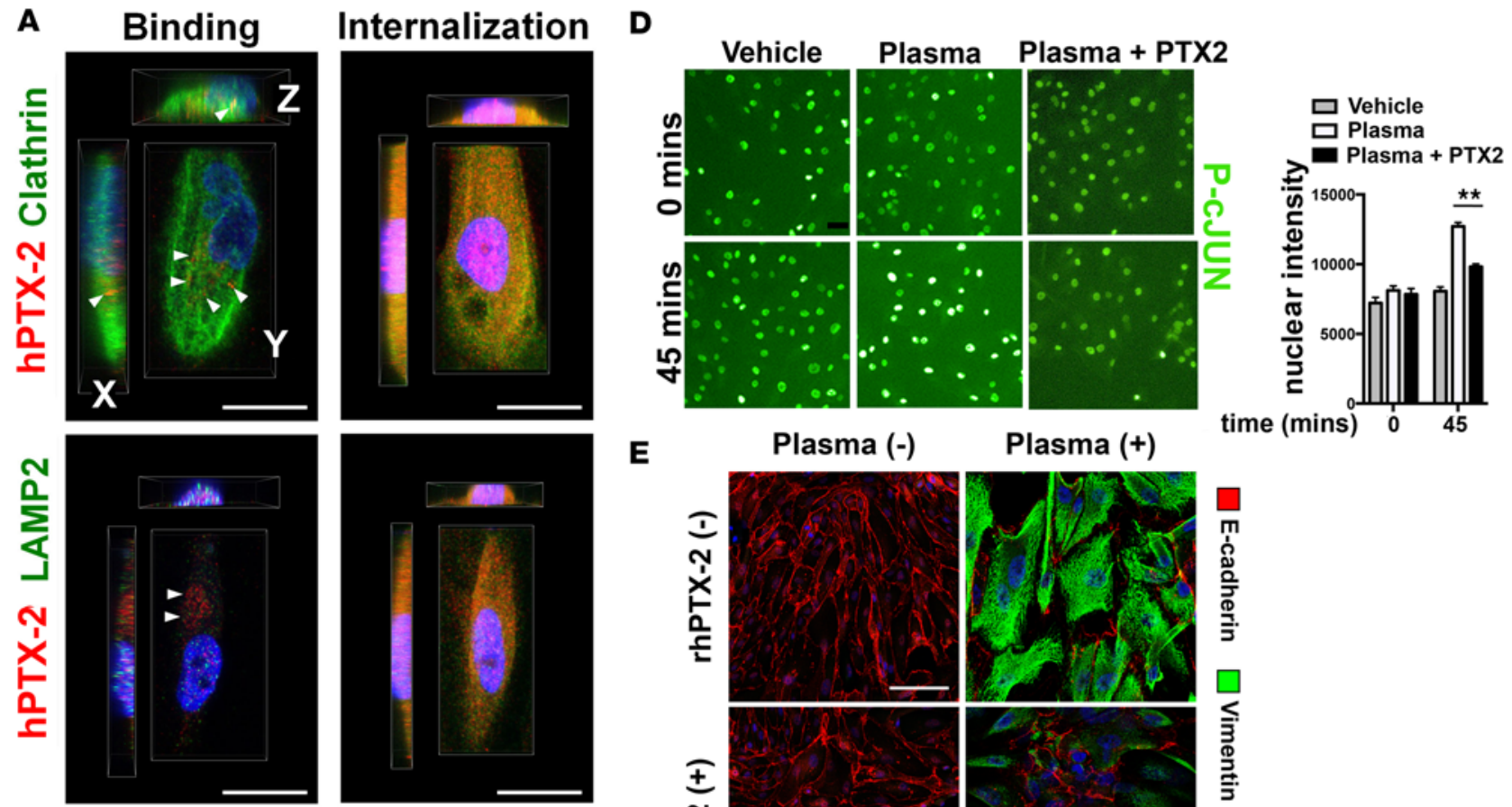

\section{E}

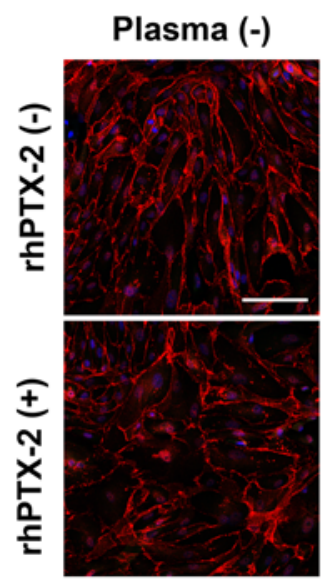

Plasma (+)
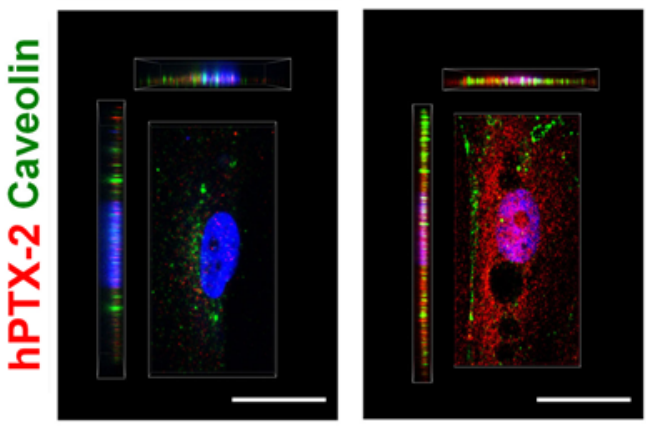

B

Human epithelial cells

PTX-2

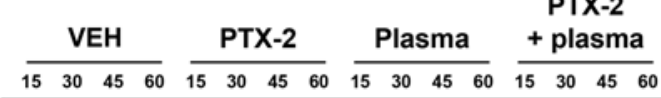

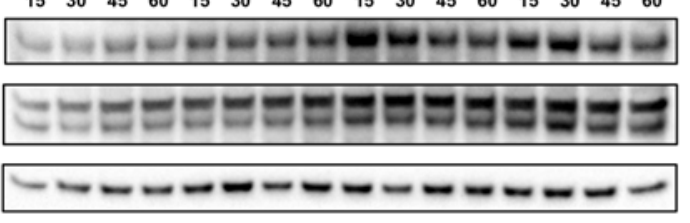

C
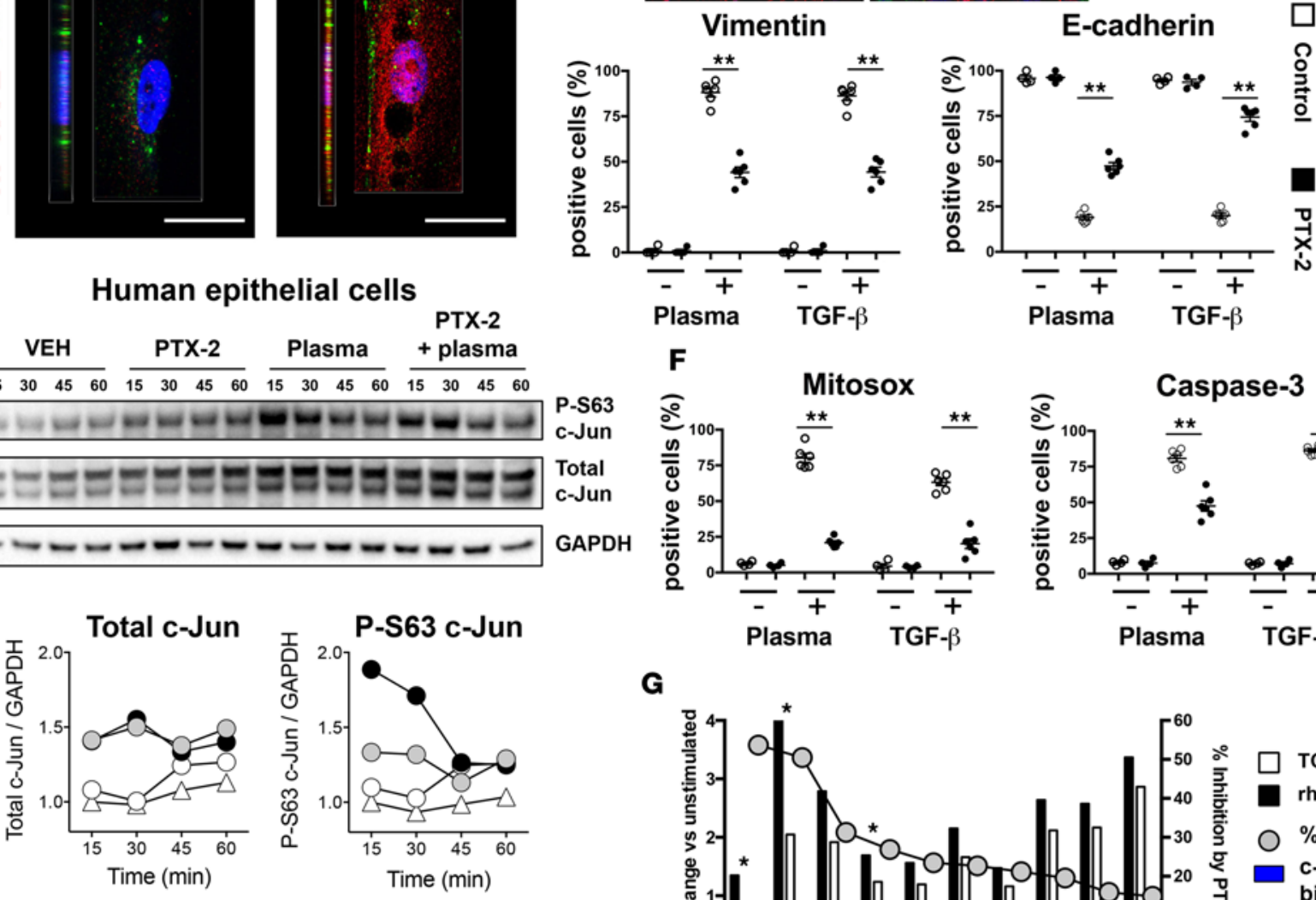

F

P-S63
C-Jun
Total
C-Jun
GAPDH

$$
\begin{array}{ll}
\triangle \text { VEH } & \begin{array}{l}
\text { Plasma } \\
\text { O PTX-2 }
\end{array} \\
\hline \begin{array}{l}
\text { PTX-2 } \\
+ \text { plasma }
\end{array}
\end{array}
$$

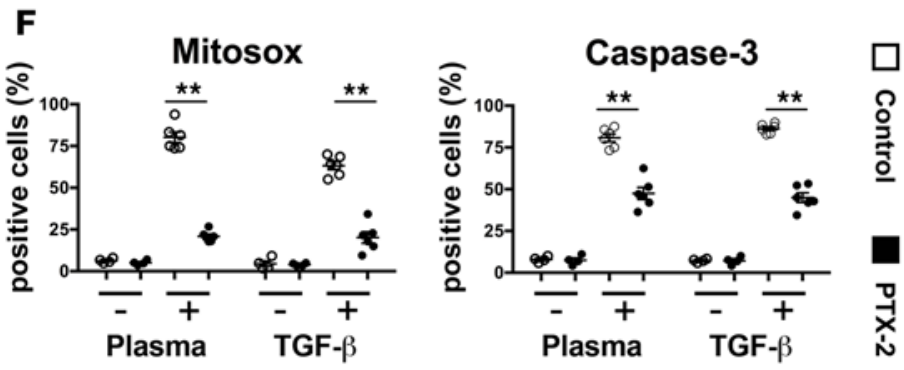

G

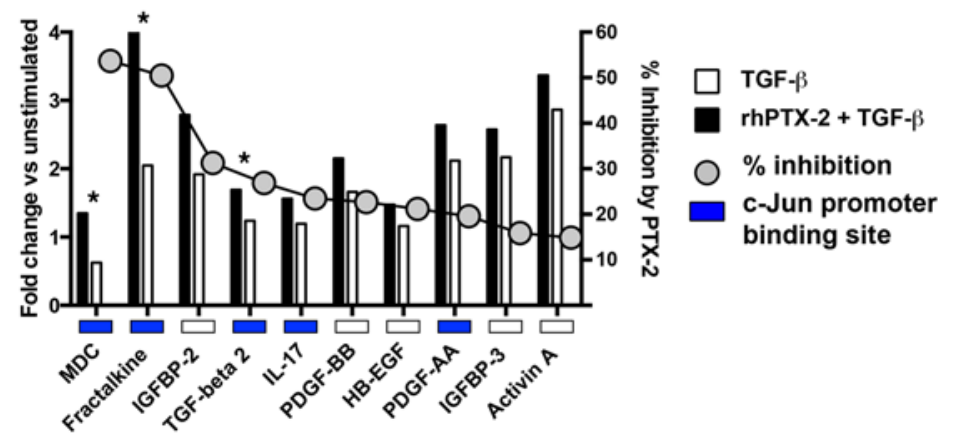


Figure 8. rhPTX-2 binds to proximal tubule epithelial cell membrane, inhibits c-Jun signaling, and protects against cellular stress. (A) $3 D$ reconstructed confocal images of representative individual human PTECs stained for rhPTX-2 (red) and clathrin, LAMP-2, or caveolin (green) showing $x, y$, and $z$ axes. The left panels indicate the surface distribution of hPTX-2 following rhPTX-2 incubation for 30 minutes on ice (arrowheads). The right panels show the distribution of internalized rhPTX-2 following incubation 30 minutes at $37^{\circ} \mathrm{C}$. (B and $\mathbf{C}$ ) Time-course Western blots showing c-Jun phosphorylation (P-S63 c-Jun) and total c-Jun levels from 15 to 60 minutes after rhPTX-2 and/or plasma shock in human fetal kidney proximal tubule epithelial cells, normalized to GAPDH. Data represent 3 experiments. (D) Images and graph quantifying nuclear p-c-JUN immunofluorescence staining intensity in human PTECs in response to plasma and PTX-2. (E) Images and quantification of E-cadherin and vimentin expression by human PTECs in resting culture conditions or in response to human plasma or TGF- $\beta 1$ for 24 hours. Note that 1 hour pretreatment of rhPTX-2 markedly blocked these responses. (F) Quantification of mitochondrial dysfunction by MitoSOX (left) and apoptosis by activated caspase-3 (right) detection 24 hours after human PTECs were challenged with human plasma or TGF- $\beta 1$, with or without 1-hour rhPTX-2 pretreatment. (C) Inhibitory effect of rhPTX-2 on factors secreted by human PTECs in response to TCF- $\beta 1$ stimulation. Fold change versus vehicle-treated PTECs and percent inhibition by rhPTX-2 are shown, and presence of c-Jun binding sites in promoters are noted. Scale bars: $25 \mu \mathrm{m} . n=3-6 /$ group. ${ }^{*} P<0.05,{ }^{* *} P<0.01$ (ANOVA with post hoc testing for multiple comparisons). VEH, vehicle.

chondrial superoxide within 24 hours, an effect that was blocked by rhPTX-2 (Figure 8F). Such release of superoxide from mitochondria is indicative of mitochondrial dysfunction, highlighted in the bioinformatics studies (Supplemental Figure 6). Exposure of PTECs to either plasma or TGF- $\beta 1$ subsequently triggered caspase-3-mediated programmed cell death, and rhPTX-2 also protected against apoptosis (Figure 8F).

The epithelium is well recognized as an important source of proinflammatory cytokines and chemokines, and therefore directly linked to inflammation. TGF- $\beta 1$ exposure resulted in enhanced secretion of 57 factors, including TNF- $\alpha$, IL-13, PDGF, EGF, IGFBP-2, IGFBP-3, CNTF, neurotrophin, activin, uPA, and thrombospondin, as detected in the epithelial secretome (Supplemental Table 4). rhPTX-2 reduced 16 of these, including factors such as MDC, fractalkine, and TGF- $\beta 2$ that can recruit and activate monocytes and macrophages, and 10 of 17 factors had AP- 1 and c-Jun binding sites within their promoters, twice the occurrence compared to genes not altered by rhPTX-2 in this experiment (Figure 8G and Supplemental Tables 4 and 5). In contrast, only one secreted factor, TIMP-3, was induced by TGF- $\beta 1$ and augmented by rhPTX-2. Although changes in their individual expression did not always achieve statistical significance due to the small sample size, collectively rhPTX-2 created a clear downward trend across most other secreted epithelial factors upregulated by TGF- $\beta 1$ ( 38 decreased versus 8 increased; data not shown). Together these findings implicate PTX-2 as a cytoprotective factor against multiple cell stress pathways, including those induced by exposure to plasma proteins and the activated form of TGF- $\beta$.

\section{Discussion}

This report demonstrates that rhPTX-2, administered systemically twice weekly, improves kidney function, reduces disease pathology, and significantly increases the lifespan of mice with a chronic and relentlessly progressive kidney disease. rhPTX-2 represents a potential approach for chronic inflammatory diseases with fibrosis, including Alport nephropathy, and potentially for other forms of CKD. The findings here reemphasize the potent effect of PTX-2 on inhibiting M1 activation of macrophages while promoting their beneficial reparative functions (Supplemental Figure 7). We also demonstrate a direct role for PTX-2 in preserving epithelial function in the setting of disease. The mechanisms by which PTX-2 exerts its benefits have not been fully elucidated $(2,7,8,11,13-15,24)$. However, PTX-2 alters the response of phagocytes to a wide range of stimuli by binding to antibody receptor complexes. The in vitro experiments presented here, and a recent study in the lung (12), indicate that epithelial cells can express different pattern recognition receptor(s) that bind directly to PTX-2. Using unbiased systems biology and cell assay-based approaches, we identified c-Jun as an important transcriptional regulator targeted by the activity of PTX-2. Together these results suggest that PTX-2 acts on both monocytes and epithelium to regulate c-Jun- and AP-1-dependent signaling and modulate cellular responses to injury and inflammatory cytokines (Supplemental Figure 7).

Current and previous strategies to design and develop therapeutics for kidney diseases favor reducing blood pressure and targeting overactivation of the immune response $(19,39,40)$. Macrophages in chronically diseased tissue can drive disease progression by provoking local tissue injury and/or stimulating excessive fibrogenic responses; on the other hand, macrophages are also adept at removing damaged cells, debris, and matrix and stimulating reparative or regenerative responses in other cells without causing local damage $(19,40-43)$. In most tissues, it appears that both deleterious and beneficial macrophages coexist, but reliable methods to confidently differentiate between them have been challenging to find. In these studies we used a costimulatory receptor (CD86), myeloid pattern recognition receptor (Trem1), and iNOS as markers of M1-type activated macrophages, whereas we selected the mannose receptor CD206 as a 
marker of cells that, in this context, promote more reparative functions (loosely referred to as M2) (44). In the Alport kidney interstitium, as well as glomerular tuft, rhPTX-2 therapy reduced the total number of macrophages. However, this reduction was restricted to $\mathrm{CD} 86^{+}$macrophages, suggesting PTX-2 selectively reduces M1-type macrophage activation in the diseased kidney and yet permits macrophages that perform beneficial functions to persist. This finding is consistent with previous studies of PTX-2 function, which have shown that by binding to Fc $\gamma$ receptors, PTX-2 triggers antiinflammatory responses in macrophages $(2,7,13,15,24,45,46)$. Importantly, PTX-2 does not simply "switch off" macrophages, but rather limits their capacity to generate proinflammatory cytokines and chemokines while also enhancing their capacity to generate antiinflammatory factors in response to a wide range of stimuli. In agreement with this finding, transcriptional analysis of whole kidney shows that rhPTX-2 reduces inflammation and immunity in Alport kidneys, and enhances certain tissue remodeling genes associated with repair, including osteopontin, MMP14, MMP2, and cathepsin-L. Tissue IL-10 levels only trended upward, in contrast to other studies in which increased IL-10 signified reparative macrophage functions stimulated by PTX-2 $(2,13)$. This difference may reflect the chronicity of the disease model studied here, and the reduction in the total macrophage population could mask increased IL-10 transcription per cell, although the lack of significance was dictated by a single outlier, and so IL-10 enhancement within the tissue in response to PTX-2 remains a potential part of the mechanism. More critically, although we identify a shift in macrophage activation states, changes in macrophage inflammatory signaling and function fall short of explaining the overall therapeutic impact of rhPTX-2 in the tissue.

Although PTX-2 has been reported to exert antifibrotic responses in many different organs by reprogramming myeloid lineage cells, including macrophages and fibrocytes, this report sheds light on additional PTX-2 homeostatic functions $(2,6,7,47)$. Previous studies indicated that PTX-2 is reduced in chronic diseases states of kidney and lung and that it binds to dying or dead cells, thereby aiding their clearance by phagocytes in an antiinflammatory way $(2,45,46)$. Therefore, the in vivo effects of PTX-2 on other cell types have been entirely and indirectly attributed to changes in leukocyte responses. Yet in the chronically diseased kidney, we identified rhPTX-2 within proximal tubular epithelium, and these PTX-2-binding epithelial cells appeared healthy. When human proximal tubular cells were cultured in serum-free media with rhPTX-2, we observed a marked cytoprotective and antiinflammatory effect in response to a single stress-inducing cytokine (active TGF- $\beta 1$ ) or a pleiotropic cell activator (plasma). This protective effect on the epithelium was seen both when cells were interrogated for mitochondrial and metabolic dysfunction, and also when evaluated for inflammatory responses or mesenchymal phenotypic changes in vitro. Leak of plasma proteins including albumin into the tubule lumen as a result of glomerular injury is increasingly implicated as one of the factors that drives tubular dysfunction in kidney disease $(48,49)$. Since PTX-2 is not normally found in urine, it is likely that in the mouse model of Alport kidney disease, glomerular injury allows PTX-2 to enter the tubular lumen (similarly to albumin), where PTX-2 interacts with the tubule cell surface. Alternatively, injury to peritubular capillaries could permit PTX-2 to interact with the basolateral surface of the epithelium.

PTX-2 had broad inhibitory effects at a transcriptional level against the activity of plasma on proximal tubule cells as well as recombinant TGF- $\beta$ in serum-free cultures, ruling out that rhPTX-2 acts on epithelial cells only indirectly as a soluble trap for bioactive factors. Because these effects of PTX-2 could not be explained by passive mechanisms, we hypothesized that PTX-2 interacts directly with an epithelial cell surface receptor (Supplemental Figure 7). We identified clear binding activity to human proximal epithelial cells and evidence to support receptor-mediated internalization into an endosomal compartment. Epithelial cells do not express Fc $\gamma$ Rs, the only receptors for PTX-2 currently known, suggesting a novel binding partner. A report has recently identified one new PTX-2 receptor expressed by lung epithelium as well as leukocytes, the C-type lectin DC-SIGN (and its nearest homolog, SIGN-R1, in mice), and also deduced that other unidentified receptors exist (12). We did not identify expression of DC-SIGN or its ortholog expressed by epithelium of the kidney, suggesting that an unidentified receptor for PTX-2 is present on the proximal epithelium, potentially in the Siglec family.

The bioinformatics analyses of whole tissue did not identify a single receptor signaling pathway that PTX-2 either activated or blocked. Rather, PTX-2 had broad antiinflammatory activity, inhibited extracellular matrix organization, and preserved mitochondrial metabolic functions. The effect on individual genes was rather modest, as has been documented for other effective therapies (21). Although most of the genes regulated by PTX-2 were involved in enhancing mitochondrial and metabolic functions, we postulate that PTX-2 acts predominantly to inhibit inflammatory pathways and that the enhanced metabolic and 
mitochondrial functions are secondary consequences. One striking observation to support this is that the gene sets enriched by PTX-2 did not include any transcriptional regulators of mitochondria or metabolism. Instead, PTX-2 strikingly suppressed transcription factors and their activators associated with inflammation. This was not restricted to a single pathway but involved suppression of several categories of intracellular signaling pathways, including many MAPKs, important components of NF- $\mathrm{KB}$ and Toll-like receptor signaling, and JAK, STAT, and SOCS proteins, all implicated in promoting chronic disease $(19,50,51)$. Although treatment with PTX-2 had modest effects on the kidney transcriptome, it nevertheless significantly enhanced overall survival. We have observed modest transcriptional changes with other therapies (21), suggesting that effective therapies have networked effects on the transcriptome in chronic diseases such as Alport nephropathy.

Despite this broad inhibitory effect on many proinflammatory pathways, the AP-1 signaling pathway is distinguished by being dominantly suppressed in whole tissue by PTX-2, below baseline levels. Three different bioinformatics analyses of all the genes suppressed by PTX-2 in disease pointed to c-Jun and AP-1 complexes as central to the overall suppression. In vitro studies of human monocytes and primary human epithelium showed that PTX-2 exposure attenuated c-Jun and AP-1 signaling, whereas it had little direct impact on RelA NF-кB p65 signaling. rhPTX-2 inhibited MAPK-dependent phosphorylation of c-Jun at a key activation site more strongly in epithelial cells, while in monocytes rhPTX-2 also reduced total c-Jun levels. In monocytes, luciferase reporter assays showed strong suppression of AP-1 signaling by PTX-2. It is noteworthy that c-Jun has been strongly implicated in the progression of many diseases, particularly through forming complexes with Fos and Fosl2 $(32,52,53)$, but direct targeting of Jun or its upstream MAPKs has proved pharmacologically challenging $(53,54)$.

It is also notable that $\mathrm{C} / \mathrm{EBP} \beta$ was consistently increased by the actions of PTX-2. Although C/EPB $\beta$ is involved in inflammation, it has been reported to play little role in M1 macrophage activation, but a more critical role in accumulating M2 macrophages and the activation of antiinflammatory pathways, including expression of IL-10, arginase-1, and soluble IL-13 decoy receptor (34). Previous studies have shown that when bound to its ligands, PTX-2 weakly activates Fc $\gamma$ Rs $(2,13,38)$. This results in partial phosphorylation of the intracellular immunoreceptor tyrosine-based activation motif (ITAM) signaling domains and is associated with inhibitory signaling. Studies in macrophages suggest there is crosstalk between $F_{c} \gamma R$, C-type lectin, and other receptors, which signal via ITAM signaling domains, and other signaling pathways such as TLR signaling, potentially through the action of the phosphatase SHP1 but also indirectly though NFAT, which activates phosphatases and transcriptional repressors (Supplemental Figure 7) $(2,50,51)$. In other settings, Fc $\gamma$ R-mediated ITAM signaling suppresses gp130 receptor-dependent signaling (which includes IL-6), as well as interferon signaling (2, 50, 51). The known roles of PTX-2 in regulating Fc $\gamma \mathrm{R}$ signaling may therefore go some way to explain the inhibitory effect on AP-1 signaling. Although epithelial cells and macrophages do not share the same ITAM domain receptors, an equivalent signaling system in the injured epithelial cell through a distinct receptor might regulate sensitivity to TLR ligation and activation of other cytokine receptors or stress-induced kinase signaling pathways.

A combination of effects may explain why attenuation of c-Jun activation by rhPTX-2 broadly impacts inflammatory, metabolic, mitochondrial, and remodeling gene sets. First, diminished c-Jun activation could directly decrease the expression of genes for which transcription relies on c-Jun binding to their promoters (31-33). AP-1 binding sites are abundant in the rhPTX-2-dependent differentially expressed gene sets, supporting this possibility. Second, the 3 Jun, 4 Fos, and 19 other AP-1 extended family members vary in their transcriptional activities, binding partners, and abundance, and different combinations of AP-1 homo- and heterodimer complexes can compete with or inhibit one another $(32,33,52)$. Therefore, altering c-Jun likely perturbs the aggregate role of all AP-1-dependent genes, including reversing AP-1-mediated transcriptional repression $(31,52,53)$. Third, because c-Jun can bind transcription factor partners outside the AP-1 family, c-Jun can control expression of a wide range of genes whose promoters lack a c-Jun binding site or recruit key transcription factors to promoters without their binding sites (29-31). For example, IL6 promoter activity requires coordinated c-Jun and NF- $\mathrm{KB}$ activation, but if c-Jun can bind to its proximal AP-1 site, no NF- $\kappa$ B binding site is needed (55). Finally transgenic overexpression of AP-1 component Fos11 (Fra1) or Fosl2 (Fra2) under a ubiquitous promoter has been shown to be sufficient to cause spontaneous inflammatory and fibrotic disease of lung, skin, liver, and upper gastrointestinal tract $(56,57)$. In these studies leukocyte and epithelial overactivation of AP1 complexes were strongly implicated in driving the fibrotic disease, similarly to our findings in the $\mathrm{Col}_{4} 3^{-/-}$kidney. 
Although we have clearly identified c-Jun and AP-1 signaling as important direct targets for PTX-2 modulation, and have excluded NF- $\mathrm{BB}$ p65 as a direct target of PTX-2 immunomodulation, it is possible that PTX-2 directly impacts additional signaling pathways including JAK/STAT and p38 MAPK. Further studies will be required to determine the direct impact of PTX-2 on signaling through these intermediate kinases.

Another member of the pentraxin family known as PTX-3 has also been implicated in kidney diseases. PTX-3 is secreted by intrinsic cells of the kidney in response to injury and locally activates the classical complement cascade. It also plays roles in clearance of apoptotic cells, but overall plays a mixed role in the pathogenesis of kidney disease, and its mechanism of action is distinct (58-60).

In conclusion, rhPTX-2 administration is an effective therapy to counteract the progression of Alport nephropathy and enhance survival in mice. PTX-2 inhibits macrophage activation in the kidney but also has antiinflammatory and cytoprotective effects directly on the proximal tubular epithelium that restore tubular cell functions. Activation of c-Jun and the AP-1 transcriptional complex are directly inhibited by the activity of PTX-2 in the kidney.

\section{Methods}

All materials were from Sigma-Aldrich unless otherwise stated.

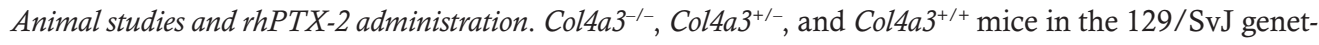
ic background were obtained from heterozygous breeding from the SJL background to the $129 \mathrm{X} 1 / \mathrm{SvJ}$ for 9 generations $(61,62)$. To mitigate minor genetic variations, animals from the same litter were utilized for different treatments. In some experiments $\mathrm{Col}_{4 a 3^{-1}}$ mice were used on the pure 129/Sv background (129-Col4a3tm1Dec/J). rhPTX-2 (PRM-151) was formulated in P5SP vehicle (10 mM sodium phosphate, $5 \%$ [wt/vol] sorbitol, $0.01 \%$ [wt/vol] polysorbate 20, $\mathrm{pH} 7.5)$ (Promedior). Mice received intraperitoneal injections of rhPTX-2 (10 mg/kg) or vehicle beginning 24 days (3.5 weeks) after birth, with daily injections for the first 3 days and thereafter twice weekly injections ending at 6 or 9 weeks. For survival studies, mice were treated twice weekly until 14 weeks after birth.

Histology staining and evaluation. Kidneys were resected after systemic perfusion with ice-cold PBS. Paraffin-embedded sections of kidney fixed with neutral-buffered formalin were used for periodic acid-Schiff (PAS), methenamine silver, and picrosirius red staining. Sclerosis index was evaluated as described previously (63). Briefly, the extent of sclerosis in 30-40 sequential glomeruli per animal in silver-stained kidney sections was determined (scarring plus capillary loop destruction) and scored by evaluators blinded to the experimental protocol as grade 0 , none; $1,0 \%-25 \% ; 2,25 \%-50 \% ; 3,50 \%-75 \%$; or $4, \geq 75 \%$. Crescents were defined and quantified as described previously (64). Interstitial fibrosis was quantified in picrosirius red-stained paraffin sections as described (65). Epithelial injury was quantified in PAS-stained kidney sections as described (66). Tissues were collected at 9 weeks for EM, prepared, and processed using standard methods as described (67). Grids were scanned using a Philips 410 electron microscope. GBM thickness measurement was performed as described previously (68). Briefly, micrographs were taken from different peripheral capillary loops of 2 distinct glomeruli. The thinnest uniform, not obliquely sectioned, areas of GBM were chosen for measurement. Four equidistant measurements were made on each capillary loop between the epithelial and endothelial plasma membrane. An arithmetic mean was calculated for 16 measurements on each glomerulus.

Immunofluorescence staining and evaluation. Mouse tissues and human cells were prepared and stained as described previously $(21,69)$. Briefly, kidneys were fixed in PLP solution for 2 hours and washed in 18\% sucrose solution overnight prior to cryopreservation and cryosectioning $(7 \mu \mathrm{m})$. For antigen detection by fluorescence, primary antibodies against the following proteins were used for immunolabeling: $\alpha$-SMA-Cy3 (1:200, clone 1A4, Sigma-Aldrich), F4/80 (1:300, Life Technologies), CD31 (1:300, BD), WT-1 (1:200, Santa Cruz Biotechnology Inc.), hSAP (PTX-2) (1:500, Abcam), CD11b (1:200, eBioscience), CD86-FITC (1:100, Abcam), CD206-FITC (1:100, BioLegend), fluorescein-labeled LTL (1:200, Vector Laboratories), vimentin (1:200, Abcam), E-cadherin (1:200, R\&D systems), cleaved caspase-3 (1:200, Cell Signaling Technology), clathrin (1:200, Abcam), LAMP-2 (1:200, Abcam), caveolin (1:200, Abcam), RelA NF-кB p65 (1:400, Cell Signaling Technology). Primary antibodies were detected with fluorescence-conjugated, affinity-purified secondary antibody labeling (1:400-1:800, Jackson ImmunoResearch Laboratories Inc.). Samples were colabeled with DAPI and mounted with ProLong Gold. Image capture and processing were carried out as previously described using confocal imaging (70). Detection of apoptotic cells was performed using the in situ cell death TUNEL detection kit (Roche) on paraffin-embedded sections, and quantification was performed as described (71). In brief, morphometry was performed by measuring percent area of positive fluorescence stain 
in 10 cortical interstitial fields sequentially selected at $\times 200$ magnification per slide using Image $\mathrm{J}$ software $(\mathrm{NIH})$. Capillary fluorescence was analyzed in images at $\times 400$ magnification captured from CD31-stained sections of 10 different fields from 6 different animals as described previously (72). Each image was divided into 266 squares by a grid. To calculate PTC density, each square containing CD $31^{+}$region resulted in a positive score. The final PTC density index was represented as a percentage of positive score.

Quantitative RT-PCR. Total RNA was extracted using TRIzol. Purity was determined by A260 to A280. cDNA was synthesized using oligo(dT) and random primers (iScript, Bio-Rad). Quantitative RT-PCR (Q-PCR) was performed using an ABI machine (7900 HT, Applied Biosystems), and iTaq Universal SYBR Green Supermix (Bio-Rad) using methods as described (71). For some experiments an initial reaction was performed to anneal polyA sequences to all RNA (polyA tailing), and then cDNA was generated using conventional Superscript II RT and a universal primer (Life Technologies). The specific primer pairs used in Q-PCR were tested for specificity and are listed in Supplemental Table 6. Gapdh was used as the housekeeping gene.

Protein isolation and Western blotting. Kidneys and cultured cells were lysed in ice-cold RIPA cell lysis buffer (Boston Bioproducts) including HALT protease and phosphatase inhibitor cocktail (Thermo) with 1 mM PMSF, using the Omni Bead Ruptor to homogenize tissue. Samples were centrifuged for 10 minutes at $14,000 \mathrm{~g}$, and the supernatant was taken for protein determination. Cell extracts containing 10-25 $\mu \mathrm{g}$ protein were prepared in SDS sample buffer and subjected to SDS-PAGE. After transfer to nitrocellulose paper and blocking, immunostaining was performed in TBST buffer. The following primary antibodies were used at 1:1000 dilution to detect the specific protein: c-Jun (60A8), p-S63-c-Jun (54B3), RelA (D14E12), p-S536 RelA (93H1), Cell Signaling Technology Inc.; C/EBP $\beta$ (E299), Abcam; and GAPDH (1:5000, 6C5), Life Technologies. Bound primary antibodies were labeled with HRP-conjugated anti-rabbit or anti-mouse IgG antisera (GE Healthcare). Bands were detected by enhanced chemiluminescence (ECL, Pierce; SuperSignal West Dura, Thermo Scientific) as recommended by the manufacturers, and luminescence was captured by the ChemiDoc MP Imaging System (Bio-Rad). For monocyte and epithelial experiments, membranes were stripped with Restore (Thermo Scientific) and reprobed to detect phosphorylated c-Jun or RelA, then total protein, then GAPDH.

Microarray experiments and bioinformatics analyses. Total RNA was extracted from mouse kidney homogenates and human PTECs using an RNeasy Mini Kit, per the manufacturer's instructions (QIAGEN). RNA integrity was confirmed using an Agilent 2100 Bioanalyzer (Agilent Technologies). High-quality RNA samples were converted to cDNA and biotin labeled using Ambion's Illumina TotalPrep RNA Amplification Kit (Life Technologies). Labeled cRNAs were hybridized to either MouseWG-6 v2 or HumanHT-12 v4 Expression BeadChips (Illumina) and imaged using an Illumina iScan system. Microarray data underwent variance stabilization and quantile normalization using the Bioconductor package lumi (73). Detailed microarray information including access to raw data, meeting Minimum Information About a Microarray Experiment (MIAME) requirements, has been deposited in the NCBI's Gene Expression Omnibus (GEO GSE85409).

PCA, a method to produce low-dimensional projection of high-dimensional data, was performed based on gene expression variability across all 12 samples from the animal experiments $\left(\right.$ Col $_{4} 3^{+/+}$,

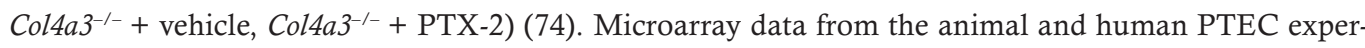
iments were separately imported into the GSEA program (http://software.broadinstitute.org/gsea) and analyzed based on approximately 3000 curated pathways (KEGG, Biocarta, Reactome, Pathway Interaction Database, etc.) and ontologies (GO) $(26,27)$. We used a false discovery rate (FDR) cutoff $<5 \%$ to identify significantly enriched gene sets. We performed leading-edge analysis to identify genes with the largest contribution to pathway enrichment (75). Leading-edge gene members of functionally related pathways were clustered using unsupervised hierarchical clustering based on their expression patterns across conditions (74). The GSEA-identified leading-edge subset of differentially expressed genes was also uploaded to the GeneGo knowledge base (Thomson Reuters) for pathway enrichment (FDR $<0.05$, Benjamini-Hochberg, hypergeometric $P$ value) and manually scored for pathway components, and also uploaded to the Search Tool for the Retrieval of Interacting Genes/Proteins (STRING, http://string-db. $\mathrm{org} /$ ) (28) to compute interactions between the core altered gene sets.

Kidney function evaluation. Urine albumin concentration was measured using an Albuwell M kit (Exocell). Urine creatinine was measured using Creatinine Liquid Reagents Assay (DIAZYME). Urea and nitrogen levels in the serum were detected by BUN reagent set (Pointe Scientific).

Cell culture and cell culture assays. Human iPSC-derived monocytes were generated from the pluripotent stem cell line HS16 originating from a 40-year-old male healthy donor. Absence of chromosomal abnormali- 
ties was confirmed by karyotype analysis. Lack of mycoplasma contamination was confirmed by MycoAlert Mycoplasma Detection Kit (LT07-218, Lonza). Pluripotent cells were maintained in mTESR1 media (05850, Stemcell Technologies) on Growth Factor Reduced Matrigel (356230, BD Biosciences). Differentiation was initiated by culturing in Stemline II Hematopoietic Stem Cell Expansion Medium (S0189, Sigma-Aldrich), 1xITS-G (41400-045, Thermo Fisher), 2 mM L-glutamine (25030-081, Thermo Fisher), 20 ng/ml bFGF (13256-029, Thermo Fisher), and $100 \mathrm{ng} / \mathrm{ml}$ human BMP-4 (120-05ET, Peprotech) for 5 days. Media was changed to Stemline II, 1xITS-G, $20 \mathrm{ng} / \mathrm{ml}$ bFGF, $100 \mathrm{ng} / \mathrm{ml}$ VEGF (100-20, Peprotech), and $100 \mathrm{ng} / \mathrm{ml}$ SCF/c-kit ligand (255-SC, R\&D Systems). On day 7, media was changed to StemPro-34 SFM, 2 mM L-glutamine, $50 \mathrm{ng} / \mathrm{ml} \mathrm{SCF} / \mathrm{C}-k i t$ ligand, $50 \mathrm{ng} / \mathrm{ml} \mathrm{IL}-3$ (200-03, Peprotech), $50 \mathrm{ng} / \mathrm{ml}$ M-CSF (300-25, PeproTech), $5 \mathrm{ng} / \mathrm{ml}$ thrombopoietin (300-18, Peprotech), and $50 \mathrm{ng} / \mathrm{ml}$ Flt-3 ligand (308-FK, R\&D Systems). On day 16, media was changed to StemPro-34 SFM, 2 mM L-glutamine, 70 ng/ml M-CSF, 70 ng/ml Flt-3 ligand, and $35 \mathrm{ng} / \mathrm{ml}$ GM-CSF, then half this media was replaced every 4 days until day 45 . Non-adherent iPS monocytes released in the media were drawn off and washed 5 minutes with PBS (10010-023, Thermo Fisher) with 2 mM EDTA (R1021, Thermo Fisher). Flow cytometry analysis for CD14 (347493, BD Biosciences) and CD45 (967217, R\&D Systems) showed $>85 \% \mathrm{CD} 14^{+} \mathrm{CD} 45^{+}$cells and no expression of $\mathrm{B}$, T, or NK cell lineage markers (CD3/14/19/20/56 anti-human Lineage Cocktail 348701, BioLegend).

iPSC-derived monocytes were cultured in XVIVO-15 media (Lonza) without serum for 16-24 hours, washed, pretreated for 15 minutes with $25 \mu \mathrm{g} / \mathrm{ml} \mathrm{rhPTX}-2$ (R\&D Systems), and stimulated for 15-60 minutes with $25 \mathrm{ng} / \mathrm{ml} \mathrm{rhTNF}-\alpha$ (PeproTech). Peripheral blood monocytes were isolated from healthy donors by negative selection and cultured $12-120$ hours with or without $5 \mu \mathrm{g} / \mathrm{ml} \mathrm{rhPTX}-2$ as described previously (2). Secreted proteins were measured by multi-analyte profiling (Rules-Based Medicine). The THP-1 AP1/ NF-кB MyD88-deficient monocytic reporter cell line (THP-1-XBlue-defMyD, InvivoGen), was used according to manufacturer's instructions. The c-Jun inhibitor SR11302 (Tocris Bioscience) was tested for toxicity in THP-1 cells and used at a dose at least 5-fold below the detection of decreased viability. The AP-1 luciferase reporter lentiviral vector (SA Biosciences, CLS-011L) contains a minimal CMV promoter and tandem repeated 5`-TGAGTCAG-3` AP-1 response elements upstream of the firefly luciferase reporter gene. THP-1 cells (ATCC) were transduced with Lipofectamine (Thermo Fisher Scientific) as instructed and selected for puromycin resistance to obtain stable reporter expression. For AP-1 transcriptional activity assays, cells were switched to unsupplemented OptiMEM media for 18-24 hours; washed and plated in OptiMEM; treated with $1-25 \mu \mathrm{g} / \mathrm{ml}$ rhPTX-2 (R\&D Systems, Novoprotein) for 60 minutes or $0.1-2 \mu \mathrm{M}$ SR11302 for 15 minutes, or vehicle controls; stimulated with 1-20 ng/ml rhTNF- $\alpha$ (Peprotech); and cultured for 18-24 hours. Secreted alkaline phosphatase was measured in culture supernatants by absorbance using QUANTI-Blue (InvivoGen), and luciferase levels were determined in cell lysates by luminescence using Bright-Glo (Promega).

Human adult PTECs and fetal epithelial cells were isolated from discarded human kidneys that were digested using a collagenase-based method as described previously (21) and purified by MACS column (Miltenyi Biotec) affinity for anti-EPCAM antibodies and LTL attached to magnetic beads. Confluent monolayers were studied and passaged up to 4 times, from at least 3 separate donors. To induce cell stress, primary PTECs, pretreated 1 hour with $25 \mu \mathrm{g} / \mathrm{ml} \mathrm{rhPTX}-2$ or human IgG (Jackson ImmunoResearch Laboratories Inc.), then challenged with $10 \%$ human plasma (Sigma-Aldrich) or $5 \mathrm{ng} / \mathrm{ml} \mathrm{rhTGF}-\beta 1$ (R\&D Systems) for 16 or 24 hours, washed with PBS, and evaluated for immunofluorescence staining, mitochondrial dysfunction using MitoSOX (Molecular Probes), and apoptosis as described (37). Supernatants were collected and cytokine concentrations measured using multiplex analysis (Raybiotech Inc.). To analyze signaling pathways, human fetal kidney epithelial cells were isolated, cultured in serum-free conditions, and activated by the same methods, then lysed and analyzed by Western blotting as described. In other experiments, PTECs were pretreated with rhPTX-2, or vehicle, followed by washing, then stimulation with $20 \%$ plasma. Epithelial cells were then fixed, permeabilized, and stained for p-c-JUN [p-S63-c-Jun (54B3), Cell Signaling Technology Inc.]. Nuclear signal intensity of p-c-JUN was quantified using Operetta CLS (PerkinElmer).

Statistics. Data are presented as mean \pm SEM, and differences between groups were assessed by ANOVA with post hoc testing and differences in survival assessed by Gehan-Breslow-Wilcoxon test, with exceptions noted in the figure legends. A $P$ value of less than 0.05 was considered significant.

Study approval. All animal studies were performed under protocols approved by the Institutional Animal Care and Use Committee of the University of Washington. Human epithelial cells were obtained from discarded tissue with written informed consent and institutional review board approval (University of Washington Medical Center, IRB447773EA; Tufts Medical Center. 


\section{Author contributions}

$\mathrm{NN}$, LB, and JSD wrote the manuscript; NN, LB, IGG, RMJ, MLL, and JSD designed the experiments; NN, LB, IGG, BGJ, AMR, and SK performed the experiments; NN, LB, IGG, BGJ, AMR, SAG, and JSD analyzed the data; and RMJ and MLL provided rhPTX-2 for in vivo experiments.

\section{Acknowledgments}

These studies were funded by: a NephCure career development grant; a gift from Promedior Inc. to the Division of Nephrology at the University of Washington; a travel grant from Asahikawa Medical University; NIH grants (DK087389, DK093493, DK094768, TR000504); AHA grant 12040023; and Biogen. We thank the Lynn and Mike Garvey Microscopy Suite for assistance with imaging; Kelly Hudkins and Charles Alpers (University of Washington) for assistance with histological evaluation; Kim Muszynski for assistance with human cell culture; Jeff Delrow and Crissa Bennett of the Fred Hutchinson Cancer Research Center Bioinformatics Core for advice and data generation; and Raghavendra Hosur for bioinformatics consultations.

Address correspondence to: Jeremy S Duffield, UW Medicine, 850 Republican Street, Box 358052, Seattle, Washington 98109, USA. Phone: 206.685.8456; E-mail: jeremysd@u.washington.edu.

1. Pilling D, Buckley CD, Salmon M, Gomer RH. Inhibition of fibrocyte differentiation by serum amyloid P. J Immunol. 2003;171(10):5537-5546.

2. Castaño AP, et al. Serum amyloid $\mathrm{P}$ inhibits fibrosis through $\mathrm{Fc}$ gamma R-dependent monocyte-macrophage regulation in vivo. Sci Transl Med. 2009;1(5):5ra13.

3. Duffield JS, Lupher ML. PRM-151 (recombinant human serum amyloid P/pentraxin 2) for the treatment of fibrosis. Drug News Perspect. 2010;23(5):305-315.

4. Botto M, et al. Amyloid deposition is delayed in mice with targeted deletion of the serum amyloid P component gene. Nat Med. 1997;3(8):855-859.

5. Bickerstaff MC, et al. Serum amyloid P component controls chromatin degradation and prevents antinuclear autoimmunity. Nat Med. 1999;5(6):694-697.

6. Pilling D, Gomer RH. Persistent lung inflammation and fibrosis in serum amyloid P component (APCs-/-) knockout mice. PLoS One. 2014;9(4):e93730.

7. Murray LA, et al. TGF-beta driven lung fibrosis is macrophage dependent and blocked by Serum amyloid P. Int J Biochem Cell Biol. 2011;43(1):154-162.

8. Cox N, Pilling D, Gomer RH. Serum amyloid P: a systemic regulator of the innate immune response. J Leukoc Biol. 2014;96(5):739-743.

9. Dillingh MR, et al. Recombinant human serum amyloid $\mathrm{P}$ in healthy volunteers and patients with pulmonary fibrosis. Pulm Pharmacol Ther. 2013;26(6):672-676.

10. Verstovsek S, et al. Role of neoplastic monocyte-derived fibrocytes in primary myelofibrosis. J Exp Med. 2016;213(9):1723-1740.

11. Lu J, Marnell LL, Marjon KD, Mold C, Du Clos TW, Sun PD. Structural recognition and functional activation of FcgammaR by innate pentraxins. Nature. 2008;456(7224):989-992.

12. Cox N, Pilling D, Gomer RH. DC-SIGN activation mediates the differential effects of SAP and CRP on the innate immune system and inhibits fibrosis in mice. Proc Natl Acad Sci U S A. 2015;112(27):8385-8390.

13. Zhang W, Xu W, Xiong S. Macrophage differentiation and polarization via phosphatidylinositol 3-kinase/Akt-ERK signaling pathway conferred by serum amyloid P component. J Immunol. 2011;187(4):1764-1777.

14. Cox N, Pilling D, Gomer RH. Distinct Fc $\gamma$ receptors mediate the effect of serum amyloid p on neutrophil adhesion and fibrocyte differentiation. J Immunol. 2014;193(4):1701-1708.

15. Du Clos TW. Pentraxins: structure, function, and role in inflammation. ISRN Inflamm. 2013;2013:379040.

16. Duffield JS. Cellular and molecular mechanisms in kidney fibrosis. J Clin Invest. 2014;124(6):2299-2306.

17. Deltas C, Pierides A, Voskarides K. Molecular genetics of familial hematuric diseases. Nephrol Dial Transplant. 2013;28(12):2946-2960.

18. Eddy AA. Overview of the cellular and molecular basis of kidney fibrosis. Kidney Int Suppl (2011). 2014;4(1):2-8.

19. Meng XM, Nikolic-Paterson DJ, Lan HY. Inflammatory processes in renal fibrosis. Nat Rev Nephrol. 2014;10(9):493-503.

20. Anker MC, Arnemann J, Neumann K, Ahrens P, Schmidt H, König R. Alport syndrome with diffuse leiomyomatosis. Am J Med Genet A. 2003;119A(3):381-385.

21. Gomez IG, et al. Anti-microRNA-21 oligonucleotides prevent Alport nephropathy progression by stimulating metabolic pathways. J Clin Invest. 2015;125(1):141-156.

22. Tanaka M, et al. Loss of the BMP antagonist USAG-1 ameliorates disease in a mouse model of the progressive hereditary kidney disease Alport syndrome. J Clin Invest. 2010;120(3):768-777.

23. Gross O, et al. Preemptive ramipril therapy delays renal failure and reduces renal fibrosis in COL4A3-knockout mice with Alport syndrome. Kidney Int. 2003;63(2):438-446.

24. Moreira AP, et al. Serum amyloid $\mathrm{P}$ attenuates $\mathrm{M} 2$ macrophage activation and protects against fungal spore-induced allergic airway disease. J Allergy Clin Immunol. 2010;126(4):712-721.e7.

25. Zhang M, et al. A phase 1 study of $\mathrm{KH} 902$, a vascular endothelial growth factor receptor decoy, for exudative age-related macu- 
lar degeneration. Ophthalmology. 2011;118(4):672-678.

26. Subramanian A, et al. Gene set enrichment analysis: a knowledge-based approach for interpreting genome-wide expression profiles. Proc Natl Acad Sci U S A. 2005;102(43):15545-15550.

27. Mootha VK, et al. PGC-1alpha-responsive genes involved in oxidative phosphorylation are coordinately downregulated in human diabetes. Nat Genet. 2003;34(3):267-273.

28. Szklarczyk D, et al. STRING v10: protein-protein interaction networks, integrated over the tree of life. Nucleic Acids Res. 2015;43(Database issue):D447-D452.

29. Bhatt D, Ghosh S. Regulation of the NF-кB-Mediated Transcription of Inflammatory Genes. Front Immunol. $2014 ; 5: 71$.

30. Oeckinghaus A, Hayden MS, Ghosh S. Crosstalk in NF-kB signaling pathways. Nat Immunol. 2011;12(8):695-708.

31. Hess J, Angel P, Schorpp-Kistner M. AP-1 subunits: quarrel and harmony among siblings. J Cell Sci. 2004;117(Pt 25):5965-5973

32. Kappelmann M, Bosserhoff A, Kuphal S. AP-1/c-Jun transcription factors: regulation and function in malignant melanoma. Eur J Cell Biol. 2014;93(1-2):76-81.

33. Shaulian E, Karin M. AP-1 in cell proliferation and survival. Oncogene. 2001;20(19):2390-2400.

34. Ruffell D, et al. A CREB-C/EBPbeta cascade induces M2 macrophage-specific gene expression and promotes muscle injury repair. Proc Natl Acad Sci U S A. 2009;106(41):17475-17480.

35. Alvarez ML, Distefano JK. The role of non-coding RNAs in diabetic nephropathy: potential applications as biomarkers for disease development and progression. Diabetes Res Clin Pract. 2013;99(1):1-11.

36. Heilig CW, et al. GLUT1 regulation of the pro-sclerotic mediators of diabetic nephropathy. Am J Nephrol. 2013;38(1):39-49.

37. Chau BN, et al. MicroRNA-21 promotes fibrosis of the kidney by silencing metabolic pathways. Sci Transl Med. 2012;4(121):121ra18.

38. Bodin $\mathrm{K}$, et al. Antibodies to human serum amyloid $\mathrm{P}$ component eliminate visceral amyloid deposits. Nature. 2010;468(7320):93-97.

39. Satou R, Gonzalez-Villalobos RA. JAK-STAT and the renin-angiotensin system: the role of the JAK-STAT pathway in blood pressure and intrarenal renin-angiotensin system regulation. JAKSTAT. 2012;1(4):250-256.

40. Adhyatmika A, Putri KS, Beljaars L, Melgert BN. The Elusive Antifibrotic Macrophage. Front Med (Lausanne). $2015 ; 2: 81$.

41. Duffield JS. Macrophages and immunologic inflammation of the kidney. Semin Nephrol. 2010;30(3):234-254.

42. Lichtnekert J, Kawakami T, Parks WC, Duffield JS. Changes in macrophage phenotype as the immune response evolves. Curr Opin Pharmacol. 2013;13(4):555-564.

43. Wynn TA, Barron L. Macrophages: master regulators of inflammation and fibrosis. Semin Liver Dis. 2010;30(3):245-257.

44. Kawakami T, et al. Resident renal mononuclear phagocytes comprise five discrete populations with distinct phenotypes and functions. J Immunol. 2013;191(6):3358-3372.

45. Bharadwaj D, Mold C, Markham E, Du Clos TW. Serum amyloid P component binds to Fc gamma receptors and opsonizes particles for phagocytosis. J Immunol. 2001;166(11):6735-6741.

46. Mold C, Baca R, Du Clos TW. Serum amyloid P component and C-reactive protein opsonize apoptotic cells for phagocytosis through Fcgamma receptors. J Autoimmun. 2002;19(3):147-154.

47. Verstovsek S, et al. Role of neoplastic monocyte-derived fibrocytes in primary myelofibrosis. J Exp Med. 2016;213(9):1723-1740

48. Guo JK, et al. Increased tubular proliferation as an adaptive response to glomerular albuminuria. J Am Soc Nephrol. 2012;23(3):429-437.

49. Abbate M, Zoja C, Remuzzi G. How does proteinuria cause progressive renal damage? J Am Soc Nephrol. 2006;17(11):2974-2984

50. Ivashkiv LB. A signal-switch hypothesis for cross-regulation of cytokine and TLR signalling pathways. Nat Rev Immunol. 2008;8(10):816-822.

51. Kang YJ, et al. Calcineurin negatively regulates TLR-mediated activation pathways. J Immunol. 2007;179(7):4598-4607.

52. Ponticos M, et al. Failed degradation of JunB contributes to overproduction of type I collagen and development of dermal fibrosis in patients with systemic sclerosis. Arthritis Rheumatol. 2015;67(1):243-253.

53. Ye N, Ding Y, Wild C, Shen Q, Zhou J. Small molecule inhibitors targeting activator protein 1 (AP-1). JMed Chem. 2014;57(16):6930-6948.

54. Koch P, Gehringer M, Laufer SA. Inhibitors of c-Jun N-terminal kinases: an update. J Med Chem. 2015;58(1):72-95.

55. Xiao W, Hodge DR, Wang L, Yang X, Zhang X, Farrar WL. NF-kappaB activates IL-6 expression through cooperation with c-Jun and IL6-AP1 site, but is independent of its IL6-NFkappaB regulatory site in autocrine human multiple myeloma cells. Cancer Biol Ther. 2004;3(10):1007-1017.

56. Kireva T, et al. Transcription factor Fra-1 induces cholangitis and liver fibrosis. Hepatology. 2011;53(4):1259-1269.

57. Eferl R, et al. Development of pulmonary fibrosis through a pathway involving the transcription factor Fra-2/AP-1. Proc Natl Acad Sci U S A. 2008;105(30):10525-10530.

58. Rovere $\mathrm{P}$, et al. The long pentraxin PTX3 binds to apoptotic cells and regulates their clearance by antigen-presenting dendritic cells. Blood. 2000;96(13):4300-4306.

59. Speeckaert MM, Speeckaert R, Carrero JJ, Vanholder R, Delanghe JR. Biology of human pentraxin 3 (PTX3) in acute and chronic kidney disease. J Clin Immunol. 2013;33(5):881-890.

60. Bassi N, et al. PTX3, Anti-PTX3, and Anti-C1q Autoantibodies in Lupus Glomerulonephritis. Clin Rev Allergy Immunol. $2015 ; 49(2): 217-226$.

61. Cosgrove D, et al. Collagen COL4A3 knockout: a mouse model for autosomal Alport syndrome. Genes Dev. 1996;10(23):2981-2992

62. Kang JS, et al. Loss of alpha3/alpha4(IV) collagen from the glomerular basement membrane induces a strain-dependent isoform switch to alpha5alpha6(IV) collagen associated with longer renal survival in Col4a3-/- Alport mice. J Am Soc Nephrol. 2006;17(7):1962-1969.

63. Ryu M, Mulay SR, Miosge N, Gross O, Anders HJ. Tumour necrosis factor- $\alpha$ drives Alport glomerulosclerosis in mice by promoting podocyte apoptosis. J Pathol. 2012;226(1):120-131.

64. Janssen U, et al. Improved survival and amelioration of nephrotoxic nephritis in intercellular adhesion molecule-1 knockout mice. J Am Soc Nephrol. 1998;9(10):1805-1814.

65. Humphreys BD, et al. Fate tracing reveals the pericyte and not epithelial origin of myofibroblasts in kidney fibrosis. Am J Pathol. 
2010;176(1):85-97.

66. Ren S, et al. LRP-6 is a coreceptor for multiple fibrogenic signaling pathways in pericytes and myofibroblasts that are inhibited by DKK-1. Proc Natl Acad Sci U S A. 2013;110(4):1440-1445.

67. Alpers CE, Hudkins KL, Pritzl P, Johnson RJ. Mechanisms of clearance of immune complexes from peritubular capillaries in the rat. Am J Pathol. 1991;139(4):855-867.

68. Das AK, Pickett TM, Tungekar MF. Glomerular basement membrane thickness - a comparison of two methods of measurement in patients with unexplained haematuria. Nephrol Dial Transplant. 1996;11(7):1256-1260.

69. Campanholle G, Ligresti G, Gharib SA, Duffield JS. Cellular mechanisms of tissue fibrosis. 3. Novel mechanisms of kidney fibrosis. Am J Physiol, Cell Physiol. 2013;304(7):C591-C603.

70. Lin SL, Kisseleva T, Brenner DA, Duffield JS. Pericytes and perivascular fibroblasts are the primary source of collagen-producing cells in obstructive fibrosis of the kidney. Am J Pathol. 2008;173(6):1617-1627.

71. Schrimpf C, et al. Pericyte TIMP3 and ADAMTS1 modulate vascular stability after kidney injury. J Am Soc Nephrol. 2012;23(5):868-883.

72. Kida Y, Ieronimakis N, Schrimpf C, Reyes M, Duffield JS. EphrinB2 reverse signaling protects against capillary rarefaction and fibrosis after kidney injury. J Am Soc Nephrol. 2013;24(4):559-572.

73. Du P, Kibbe WA, Lin SM. lumi: a pipeline for processing Illumina microarray. Bioinformatics. 2008;24(13):1547-1548.

74. Saeed AI, et al. TM4: a free, open-source system for microarray data management and analysis. BioTechniques. 2003;34(2):374-378

75. Subramanian A, Kuehn H, Gould J, Tamayo P, Mesirov JP. GSEA-P: a desktop application for Gene Set Enrichment Analysis. Bioinformatics. 2007;23(23):3251-3253. 